Fields Institute Communications volume 4,1995

\title{
The Constrained Liapunov-Schmidt Procedure and Periodic Orbits
}

\author{
Martin Golubitsky* \\ Department of Mathematics \\ University of Houston \\ Houston, TX 77204-3476, USA \\ Jerrold E. Marsden** \\ Department of Mathematics \\ University of California, Berkeley \\ Berkeley, CA 94720, USA \\ Ian Stewart ${ }^{\dagger}$ \\ Mathematics Institute \\ University of Warwick \\ Coventry CV4 7AL, UK \\ Michael Dellnitz ${ }^{\ddagger}$ \\ Department of Mathematics \\ University of Hamburg \\ D-20146 Hamburg, Germany
}

\begin{abstract}
This paper develops the Liapunov-Schmidt procedure for systems with additional constraints such as having a first integral, being Hamiltonian, or being a gradient system. Similar developments for systems with symmetry, including reversibility, are well known, and the method of this paper augments and is consistent with that approach. One of the results states that the bifurcation equation for Hamiltonian systems is actually a Hamiltonian vector field. In general, we use "implicit constraints" to encode the information constraining the system. The method is applied to the Liapunov center theorem for reversible systems and systems with an integral, as well as to the Hamiltonian Hopf bifurcation and resonance bifurcations for Hamiltonian and reversible systems.
\end{abstract}

1991 Mathematics Subject Classification. Primary 58F05; Secondary 58F14, 70K30.

-Research partially supported by NSF Grant DMS-9101836, the Texas Advanced Research Program (003652037) and The Fields Institute.

* Research partially supported by NSF Grant DNS-9302992 and The Fields Institute.

'Research partially supported by The Fields Institute, the Science and Engineering Research Council of the UK, and a European Community Laboratory Twinning grant (European Bifurcation Theory Group).

tResearch partially supported by The Fields Institute, and a European Community Laboratory Twinning grant (European Bifurcation Theory Group). 


\section{Introduction}

The Liapunov-Schmidt procedure is widely used to reduce bifurcation problems that satisfy appropriate hypotheses to the solution of equations defined on finite-dimensional spaces. This procedure has been adapted to systems with symmetry (see Golubitsky and Schaeffer [1985] Chapter 7 Section 2). As observed in Hale [1969], the Liapunov-Schmidt procedure is especially effective for finding periodic orbits arising through Hopf bifurcation and may be adapted to Hopf bifurcation with symmetry (see Golubitsky, Stewart and Schaeffer [1988] and references therein).

In this paper we observe that the Liapunov-Schmidt procedure preserves additional structure, of a kind that we call an "implicit constraint". We show that this observation can be used to derive several known results (listed below) in a uniform and simple manner. The constraint is implicit in the sense that it becomes an implicit condition on the Liapunov-Schmidt reduced bifurcation equation. However, it may be an explicit "system constraint" to begin with, such as the conditions of having a first integral, being Hamiltonian, or being a gradient.

Implicit constraints can greatly simplify the solution of the Liapunov-Schmidt reduced equations because of the restrictions they impose on their form. The Hamiltonian case is especially well behaved because, under appropriate hypotheses, the reduced bifurcation equation defines a Hamiltonian vector field. In this sense the Liapunov-Schmidt procedure performs a Hamiltonian reduction analogous to the well known "orbit space" reduction method for Hamiltonians with a symmetry group; see for example Abraham and Marsden [1978].

The paper divides into three main parts:

- In Part I, we set the stage by adding the extra ingredient of a constraint to the Liapunov-Schmidt procedure for systems with symmetry. We illustrate how to use this extra ingredient by giving a new proof of the Liapunov senter theorem for systems with a first integral and extending it to an equivariant context.

- In Part II, we apply the constrained Liapunov-Schmidt procedure to give a simple and direct determination of the periodic orbit structure of the Hamiltonian-Hopf bifurcation.

- In Part III, we apply this method to study the bifurcation structure of periodic orbits near a $k: \ell$ resonance for both reversible and Hamiltonian systems.

We will give a more detailed introduction to each of the applications and discuss some of the relevant literature throughout the text.

Acknowledgments We would like to express our sincere thanks to Tom Bridges, Hans Duistermaat, Vivien Kirk, Victor LeBlanc, James Montaldi, Jan-Cees van der Meer, Tudor Ratiu, Jürgen Scheurle, Alan Weinstein, and, in particular, André Vanderbauwhede, for useful comments and discussions.

\section{Part I The Constrained Liapunov-Schmidt Procedure}

\section{Introduction}

The basic idea of the method is as follows. Particular types of solutions in a differential equation, such as a fixed point, relative equilibrium, or a periodic orbit, can be found by determining the zeros of an appropriate map and applying the 
Liapunov-Schmidt procedure. For example, the problem of finding periodic orbits can be formulated as the search for zeros of a map $F(u, \tau)=0$ near a known zero, where $u$ is an element of a loop space (a candidate periodic orbit) and $\tau$ is the perturbed period, an idea that goes back to Hale [1969]. Additionally, $F$ may depend on bifurcation parameters $\lambda$, but we suppress these for the moment. The Liapunov-Schmidt procedure leads to a reduced bifurcation equation $g(k, \tau)=0$ defined for $k$ in the kernel of the linearization of the original equation, and the reduced map $g$ inherits the symmetries of $F$. If we are looking for periodic orbits, then the map $F$ has a natural symmetry group $\mathbf{S}^{1}$ representing phase shifts along the periodic orbits, as well as any other symmetries that may be present in the original problem.

When the system under consideration has extra structure, we can often encode this structure in an identity of the form $\Phi(u, \tau, F(u, \tau)) \equiv 0$, for a suitable mapping $\Phi$. We call such an identity an implicit constraint (implicit because of the way $F$ occurs in $\Phi$ ). We show below that an implicit constraint on $F$ imposes a related implicit constraint on the reduced map $g$, so that $\varphi(k, \tau, g(k, \tau))=0$ for a function $\varphi$ derived from $\Phi$.

Our first illustration of the method is an application to the Liapunov center theorem. Here the extra structure that leads to an implicit constraint is given by a first integral. The kernel is two-dimensional and is identified with the complex numbers $\mathbb{C}_{;}$the circle group $S^{1}$ may be viewed as the unit modulus complex numbers with its usual action on $\mathbb{C}$; and $\mathbf{S}^{1}$-equivariance implies that $g: \mathbb{C} \times \mathbb{R} \rightarrow \mathbb{C}$ has the form

$$
g(z, \tau)=p\left(|z|^{2}, \tau\right) z+q\left(|z|^{2}, \tau\right) i z
$$

for real-valued functions $p$ and $q$. The first integral condition implies that $p$ and $q$ are related in such a way that $p$ vanishes if $q$ vanishes. Therefore, to find solutions, it is enough to solve the equation $q=0$. As in Hopf bifurcation, it can be shown that $q_{\tau}$ is nonzero at the origin, so we can solve for zeros by the implicit function theorem. We remark that this method using the Liapunov-Schmidt procedure together with symmetries and an implicit constraint avoids the procedure of blowing up the singularity.

If we make the stronger assumption that the system is Hamiltonian or reversible, then it can be proved directly that the function $p$ in (1.1) is identically zero. In the reversible case, this is seen directly from the fact that the map $g$ inherits the reversible symmetry structure. In the Hamiltonian case, the implicit constraint states that the map $g$ is a Hamiltonian vector field (depending parametrically on $\tau$ ), using the usual symplectic structure on $\mathbb{C}$ with the real and imaginary parts of $z$ as conjugate variables. We use this Hamiltonian structure in place of having a first integral. In fact, it is easy to see that the map $g$ in (1.1) is Hamiltonian if and only if $p=0$. Vanderbauwhede and van der Meer [1994] have also proved, independently and by a different method, that the reduced bifurcation equation inherits a Hamiltonian structure.

\section{Implicit constraints}

This section develops the constrained Liapunov-Schmidt procedure. In practice, a key step is to translate conditions such as having a first integral, being variational, or being Hamiltonian, into constraints and to pass these constraints to 
the bifurcation equation. We illustrate this procedure in various applications, but for the moment we concentrate on the abstract formulation.

Let $\mathcal{X}$ and $\mathcal{Y}$ be Banach spaces and let $F: \mathcal{X} \rightarrow \mathcal{Y}$ be a $C^{\infty}$ map whose zeros we seek. Assume that $F(0)=0$. Let $d F$ denote the (Fréchet) derivative of $F$ and assume that $d F(0)$ is Fredholm. Let $\Gamma$ be a compact Lie group acting linearly on both $\mathcal{X}$ and $\mathcal{Y}$ and suppose that $F$ is $\Gamma$-equivariant. We say that there is an implicit constraint if there is another Banach space $\mathcal{W}$ on which $\Gamma$ also acts, and a $C^{\infty}$ equivariant map

$$
\Phi: \mathcal{X} \times \mathcal{Y} \rightarrow \mathcal{W}
$$

such that

$$
\Phi(X, F(X))=0
$$

for all $X \in \mathcal{X}$ (or in a neighborhood of zero, since the constructions here are local).

For a system of ODEs, the possession of a first integral can be phrased as an implicit constraint, as we now explain. Consider a system of ODEs on $\mathbb{R}^{n}$ of the form

$$
\dot{X}=f(X)
$$

and assume that it has a first integral; that is, there exists a function $H: \mathbb{R}^{n} \rightarrow \mathbb{R}$ that is constant along trajectories:

$$
H(X(s))=H(X(0))
$$

for all solutions $X(s)$. Differentiation with respect to $s$ shows that this condition is equivalent to

$$
d H(X) \cdot f(X)=0
$$

for all $X$. Suppose that we are looking for equilibria of this system; that is, looking for zeros of $f$. Then we can choose $\mathcal{X}=\mathcal{Y}=\mathbb{R}^{n}$ and define

$$
\Phi(X, Y)=d H(X) \cdot Y
$$

and so the condition that $H$ be an integral is equivalent to

$$
\Phi(X, f(X))=0
$$

which is an implicit constraint. Looking for equilibria, while conceptually simple, is an important problem in Hamiltonian bifurcation theory, and this remark lets us investigate the basic bifurcations (Hamiltonian pitchfork, Hamiltonian saddle node, etc.). The problem of finding periodic orbits is a bit more subtle, but the basic idea is similar, as we shall see in the next section.

We now investigate the condition imposed on the bifurcation equation by an implicit constraint. Since $d F(0)$ is assumed to be Fredholm, $\mathcal{K}:=\operatorname{ker} d F(0)$ has a $\Gamma$-invariant closed complement $\mathcal{M}$ so that $\mathcal{X}=\mathcal{K} \oplus \mathcal{M}$. Similarly $\mathcal{R}:=$ range $d F(0)$ is closed and has a $\Gamma$-invariant closed complement $\mathcal{N}$, so that $\mathcal{Y}=\mathcal{R} \oplus \mathcal{N}$. Let $\mathbf{P}: \mathcal{Y} \rightarrow \mathcal{R}$ denote the projection with kernel $\mathcal{N}$ and (locally) define a $C^{\infty}$ map $w: \mathcal{K} \rightarrow \mathcal{M}$ with $w(0)=0$ by solving

$$
\mathrm{P} F(k+w)=0
$$


for $w$ as a function of $k$ using the implicit function theorem. Uniqueness of the solution $w(k)$ and invariance of the splitting under $\Gamma$ imply that $w$ is $\Gamma$-equivariant. Moreover,

$$
d w(0)=0
$$

Higher derivatives of $w$ at zero are determined, as usual, by implicit differentiation. Clearly, solutions of the equation $F(X)=0$ are given by $X=k+w(k)$, where $k$ is a solution of the bifurcation equation

$$
g(k):=(I-\mathbf{P}) F(k+w(k))=0 .
$$

To see how the extra structure (2.1) affects the bifurcation equation, define $\varphi$ : $\mathcal{K} \times \mathcal{N} \rightarrow \mathcal{W}$ by

$$
\varphi(k, n)=\Phi(k+w(k), n) .
$$

Observe that $\varphi$ is $\Gamma$-equivariant because $w$ and $\Phi$ are. The derivatives of $\varphi$ at $(0,0)$ can be calculated in terms of the derivatives of $\Phi$ and $w$. From (2.2) and (2.4), we get

$$
\begin{aligned}
\varphi(k, g(k)) & =\Phi(k+w(k),(I-\mathbf{P}) F(k+w(k))) \\
& =\Phi(k+w(k), F(k+w(k))) \\
& =0
\end{aligned}
$$

by (2.1). Thus the implicit constraint

$$
\varphi(k, g(k))=0
$$

encodes the implications for the bifurcation equation (2.4) of the extra structure in the original problem. This imposes conditions on the bifurcation equation similar to those imposed by equivariance. We will see how to deal with such conditions and how to sometimes make them explicit (for example in the Hamiltonian case) in the following sections. We begin by showing that this technique gives an easy and natural proof of the Liapunov center theorem.

\section{The Liapunov center theorem}

The Liapunov center theorem is one of the classical periodic orbit theorems for Hamiltonian systems. We give a proof of it that illustrates the method of the preceding section. We prove it for systems with a first integral, which includes the Hamiltonian case. In the Hamiltonian case there is additional structure in the bifurcation equation, but it leads to the same conclusion. As we have indicated, this extra structure states that the map $g$ is a Hamiltonian vector field, a methodology we shall deal with shortly. Expositions of the Liapunov center theorem are found in, for example, Kelley [1967] and Abraham and Marsden [1978, pages 496-499]; the latter giving a proof based on blowing up the singularity due to Duistermaat. We also note the approach of Alexander and Yorke [1978] (see also Schmidt $[1976 a, b])$ which links the Liapunov center theorem with the Hopf bifurcation. See also the proof in Vanderbauwhede [1982b]. In addition, Liapunov center theorems for reversible systems can be found in Vanderbauwhede [1982a], Sevryuk [1986] and Golubitsky, Krupa and Lim [1991].

Let $f$ be a $C^{x}$ vector field on a $C^{x}$ manifold $M$. Assume: 
(H1) $X_{0}$ is a fixed point of the system, that is, $f\left(X_{0}\right)=0$.

(H2) The linearization $A_{0}=d f\left(X_{0}\right)$ has simple eigenvalues $\pm \omega_{0} i$, and $k \omega_{0} i$ is not an eigenvalue for $k=0,2,3, \ldots$ Let $V$ denote the eigenspace associated to the eigenvalues $\pm \omega_{0} i$.

(H3) The vector field $f$ has a $C^{\infty}$ first integral $H: M \rightarrow \mathbb{R}$ (that is, $d H(X) \cdot f(X)=0$ ) with the following two properties:

(a) $d H\left(X_{0}\right) \mid V=0$;

(b) $d^{2} H\left(X_{0}\right) \mid V$ is nondegenerate.

Theorem 3.1 Under conditions (H1)-(H3) there is a one-parameter family of periodic orbits in a neighborhood of $X_{0}$ with periods close to $2 \pi / \omega_{0}$. They locally fill out an invariant $C^{\infty}$ manifold of dimension two that is tangent to $V$ at $X_{0}$.

Proof Rescaling time, we can assume that, $\omega_{0}=1$. Passing to a coordinate chart in $M$ we can assume that $M=\mathbb{R}^{n}$, since the theorem, while intrinsic for manifolds, is local. Let $\mathcal{C}_{2 \pi}^{1}$ be the space of $C^{1}$ maps $u: S^{1} \rightarrow \mathbb{R}^{n}$ and let $\mathcal{C}_{2 \pi}^{0}$ be the space of $C^{0}$ maps $v: \mathbf{S}^{1} \rightarrow \mathbb{R}^{n}$. Define

$$
F: \mathcal{C}_{2 \pi}^{1} \times \mathbb{R} \rightarrow \mathcal{C}_{2 \pi}^{0}
$$

by

$$
F(u, \tau)=(1+\tau) \frac{d u}{d s}-f(u)
$$

The map $F$ is $C^{\infty}$ by the ' $\Omega$-lemma': see, for example, Abraham, Marsden and Ratiu [1988], Section 2.4.

The group $\mathbf{S}^{1}$ acts on $\mathcal{Y}=\mathcal{C}_{2 \pi}^{0}$ by $(\theta \cdot v)(s)=v(s+\theta)$ and similarly on $\mathcal{C}_{2 \pi}^{1}$. Further, $\mathbf{S}^{1}$ acts on $\mathcal{X}=\mathcal{C}_{2 \pi}^{1} \times \mathbb{R}$ with trivial action on $\mathbb{R}$. With these actions, $F$ is $\mathbf{S}^{1}$-equivariant.

The derivative of $F$ at $u \equiv X_{0}$ is $L$, where

$$
L v \equiv d F\left(X_{0}, 0\right) \cdot v=v^{\prime}-A_{0} v .
$$

By (H2),

$$
\begin{aligned}
\operatorname{ker} L & =\operatorname{span}\left\{\operatorname{Re}\left(e^{i s} v_{0}\right), \operatorname{Im}\left(e^{i s} v_{0}\right)\right\} \\
& =\left\{\operatorname{Re}\left(z e^{i s} v_{0}\right) \mid z \in \mathbb{C}\right\}
\end{aligned}
$$

where $A_{0} v_{0}=i v_{0}$. Thus we can identify the kernel of $d F\left(X_{0}, 0\right)$ with $\mathbb{C}$ via the $\operatorname{map} z \mapsto \operatorname{Re}\left(z e^{i s} v_{0}\right)$.

Choose orthonormal coordinates on $\mathbb{R}^{n}$ so that the first two coordinates, when complexified, span the eigenspace corresponding to eigenvalues $\pm i$ and that on this space we have

$$
A_{0}=\left[\begin{array}{cc}
0 & 1 \\
-1 & 0
\end{array}\right]
$$

The components of the (complex) vector $v_{0}$ are $(1, i, 0, \ldots, 0)$. Differentiating the conservation condition $d H(X) \cdot f(X)=0$ twice with respect to $X$ and evaluating at $X=X_{0}$ shows that the matrices representing $A_{0}$ and $d^{2} H\left(X_{0}\right)$ commute. A $2 \times 2$ matrix calculation shows that on the kernel, the matrix representing $d^{2} H\left(X_{0}\right)$ is a nonzero multiple of the identity, say $d^{2} H\left(X_{0}\right)=\mu I$. 
Now we use the Fredholm alternative relative to the $L^{2}$ inner product obtained by integrating the standard inner product on $\mathbb{R}^{n}$. Let $\mathcal{M}=$ range $L^{*}$ be the orthogonal complement of $\mathcal{K}=\operatorname{ker} L$, and let $\mathcal{N}=\operatorname{ker} L^{*}$ be the orthogonal complement to $\mathcal{R}=$ range $L$. Notice that $L^{*} v=-v^{\prime}-A_{0}^{*} v$. It is important to be careful with this splitting, because $L$ and $L^{*}$ are differential operators and a derivative is lost. However these operators are in fact elliptic, so this causes no difficulties: see Golubitsky and Schaeffer [1985], p. 332 for details.

The Liapunov-Schmidt procedure with constraints, as described in the preceding section, may be applied. This procedure gives a bifurcation equation

$$
g: \mathbb{C} \times \mathbb{R} \rightarrow \mathbb{C} .
$$

Being $\mathbf{S}^{1}$-equivariant, $g$ has the form

$$
g(z, \tau)=p\left(|z|^{2}, \tau\right) z+q\left(|z|^{2}, \tau\right) i z ;
$$

see Golubitsky and Schaeffer [1985], p. 344. Note that $p(0,0)=0$ and $q(0,0)=$ 0 since the linear terms vanish in any Liapunov-Schmidt reduced map. In the reversible case $p=0$ identically, so it suffices to solve the equation $q=0$. We now show that a similar approach works for systems with a first integral; more precisely, we can use the first integral to show below that $q=0$ implies $p=0$.

In the case of Hamiltonian systems, our general results below will show that the map $g$ is a Hamiltonian vector field on $\mathbb{C}$. Since any Hamiltonian vector field on $\mathbb{C}$ has the form $f(z)=-2 i \partial H / \partial \bar{z}$ for some real valued function $H(z, \tilde{z})$, it follows that $g$ is purely imaginary, and hence $p=0$ identically (see Bridges [1990] for another argument along these lines). In this respect, the Hamiltonian and reversible cases are similar, as is well known. The resemblance is less close in other situations; see Roberts and Quispel [1992].

The calculation in Golubitsky and Schaeffer [1985], pp. 344-349, shows that

$$
q_{\tau}(0,0)=-1 \text {. }
$$

Thus, we can solve $q=0$ for $\tau=\tau\left(|z|^{2}\right)$. We claim that this automatically defines the required manifold of periodic orbits. To prove this, we must show that $\tau=\tau\left(|z|^{2}\right)$ implies $p=0$ for $z$ small. To do this we use the implicit constraint afforded by the first integral. Define

$$
\Phi: \mathcal{X} \times \mathcal{Y} \rightarrow \mathbb{R}
$$

by

$$
\Phi((u, \tau), v)=\int_{0}^{2 \pi} d H(u(s)) \cdot v(s) d s
$$

Note that

$$
\begin{aligned}
\Phi((u, \tau), F(u, \tau)) & =\int_{0}^{2 \pi} d H(u(s)) \cdot\left[(1+\tau) \frac{d u}{d s}-f(u(s))\right] d s \\
& =(1+\tau) \int_{0}^{2 \pi} \frac{d}{d s} H(u(s)) d s-\int_{0}^{2 \pi} d H(u(s)) \cdot f(u(s)) d s .
\end{aligned}
$$

The first term in (3.5) vanishes since $H(u(2 \pi))-H(u(0))=0$ by the periodicity of $u$, while the integrand in the second term vanishes pointwise because $H$ is a first 
integral of $f$ as in (H3). Thus (2.1) holds, so the bifurcation equation satisfies (2.6). In other words,

$$
\varphi((z, \tau), g(z, \tau))=0
$$

so that

$$
\Phi(z+w(z, \tau), \tau, g(z, \tau))=0
$$

whence

$$
\int_{0}^{2 \pi} d H\left(u_{z}(s)\right) \cdot g(z, \tau) d s=0 .
$$

Here $u_{z}(s)$ is the element of $\mathcal{X}=\mathcal{C}_{2 \pi}^{1}$ corresponding to the implicitly defined function $z+w(z, \tau)$ and so has the form $\operatorname{Re}\left(z e^{i s} v_{0}\right)+O\left(|z|^{2}\right)$, and $g(z)=: \zeta=$ $p\left(|z|^{2}, \tau\right) z+q\left(|z|^{2}, \tau\right) i z$. We regard $\zeta$ as an element of $\mathcal{Y}$ by the identification

$$
\zeta \mapsto \operatorname{Re}\left(\zeta e^{i s} v_{0}^{*}\right)
$$

where $v_{0}^{*}$ is the eigenvector for $A_{0}^{*}$, that is, $A_{0}^{*} v_{0}^{*}=-i v_{0}^{*}$. The first two components of $v_{0}$ and $v_{0}^{*}$ are the same.

Choose $\tau=\tau\left(|z|^{2}\right)$ so that $q=0$ as above. Then

$$
0=p\left(|z|^{2}, \tau\left(|z|^{2}\right)\right) \int_{0}^{2 \pi} d H\left(\operatorname{Re}\left(z e^{i s} v_{0}+O\left(|z|^{2}\right)\right)\right) \cdot \operatorname{Re}\left(z e^{i s} v_{0}^{*}\right) d s
$$

We show that the second factor (that is, the integral term) is nonzero for small $|z|$. To do so let $z=r$ be real and let

$$
\Psi(r)=\int_{0}^{2 \pi} d H\left(\operatorname{Re}\left(r e^{i s} v_{0}+O\left(r^{2}\right)\right)\right) \cdot \operatorname{Re}\left(e^{i s} v_{0}^{*}\right) d s .
$$

Note that $\Psi(0)=0$ and that

$$
\Psi^{\prime}(0)=\int_{0}^{2 \pi} d^{2} H(0) \cdot\left(\operatorname{Re}\left(e^{i s} v_{0}\right), \operatorname{Re}\left(e^{i s} v_{0}^{*}\right)\right) d s .
$$

By the choice of inner product,

$$
\Psi^{\prime}(0)=\mu \int_{0}^{2 \pi}\left\langle\left(\operatorname{Re}\left(e^{i s} v_{0}\right), \operatorname{Re}\left(e^{i s} v_{0}^{*}\right)\right)\right\rangle d s=2 \pi \mu
$$

and so $\Psi(r) \neq 0$ for $r>0$ and small. Identity (3.7) now implies that $p\left(|z|^{2}, \tau\left(|z|^{2}\right)\right)=$ 0 for $z$ small.

The smoothness of the manifold of periodic orbits follows from the smoothness of $\tau\left(|z|^{2}\right)$, which is guaranteed by the implicit function theorem.

\section{An equivariant Liapunov center theorem}

The proof of the Liapunov center theorem given in the previous section automatically produces an analogous equivariant theorem, whose hypotheses we now explain.

Let $\Gamma \subset \mathbf{O}(n)$ be a group acting in the usual way on $\mathbb{R}^{n}$. Assume that the vector field $f: \mathbb{R}^{n} \rightarrow \mathbb{R}^{n}$ is $\Gamma$-equivariant and that

(EI) $f\left(X_{0}\right)=0$ where $X_{0}$ is fxed by $\Gamma$. 
As before, let $A_{0}=d f\left(X_{0}\right)$ and observe that $\Gamma$-equivariance of $\int$ implies that $A_{0}$ commutes with $\Gamma$. It follows that eigenspaces of $A_{0}$ are $\Gamma$-invariant. In particular, eigenvalues of high multiplicity may be expected. Our second assumption is the following.

(E2a) The cigenvalues of $A_{0}$ include $\pm \omega_{0} i$, and $k \omega_{0} i$ is not an eigenvalue for $k=0,2,3 \ldots$.

Let $V$ denote the generalized eigenspace associated to the eigenvalues $\pm \omega_{0} i$. We replace the previous assumption that the eigenvalues $\pm \omega_{0} i$ are simple by requiring $V$ to be $\Gamma$-simple. This notion is the $\Gamma$-equivariant version of simple eigenvalues (see Golubitsky, Stewart and Schaeffer [1988]) and is defined as follows:

Definition 4.1 The action of the group $\Gamma$ on $V$ is said to be $\Gamma$-simple if either

(a) $V=W \oplus W$ and $\Gamma$ acts absolutely irreducibly on $W$, or

(b) $\Gamma$ acts irreducibly, but not absolutely irreducibly, on $V$.

Our next assumption is the following:

(E2b) $\Gamma$ acts $\Gamma$-simply on $V$.

There is an action of $\mathbf{S}^{\mathbf{l}}$ on $V$ induced by the one-parameter group $\exp \left(s A_{0}^{T}\right)$. Since $A_{0}$ commutes with $\Gamma$, so does this action of $\mathbf{S}^{\mathbf{l}}$. Thus, we have a well defined action of $\Gamma \times \mathbf{S}^{1}$ on $V$. We now determine families of periodic solutions by looking for their symmetries. 'This involves two more conditions, as follows:

(E3a) Let $\Sigma \subset \Gamma \times \mathbf{S}^{1}$ be an isotropy subgroup of the action of $\Gamma \times \mathbf{S}^{1}$ on $V$. Assume that $\operatorname{dim} \operatorname{Fix}_{V}(\Sigma)=2$.

(E3b) $f$ has a $C^{\infty}$ first integral $H: \mathbb{R}^{2 m} \rightarrow \mathbb{R}$ such that

(i) $d H\left(X_{0}\right) \mid \operatorname{Fix}_{V}(\Sigma)=0$;

(ii) $d^{2} H\left(X_{0}\right) \mid \mathrm{Fix}_{V}(\Sigma)$ is nondegenerate.

To state the main theorem, we define what is meant by the symmetry of a periodic solution. An element $\sigma=(\gamma, \theta) \in \Gamma \times \mathbf{S}^{\mathbf{1}}$ is called a symmetry of a $2 \pi$ periodic function $X(t)$ if $\gamma X(t)=X(t-\theta)$. That is, the periodic orbit is preserved as a set by $\gamma$; uniqueness of solutions to ordinary differential equations guarantees the existence of a unique $\theta$.

We may now prove an equivariant version of the Liapunov center theorem:

Theorem 4.2 Under conditions (E1)-(E3), there is a one-parameter family of periodic orbits in a neighborhood of $X_{0}$ with $\Sigma$ symmetry and periods close to $2 \pi / \omega_{0}$. They locally fill out an invariant $C^{\infty}$ manifold of dimension two that is tangent to $\operatorname{Fix}_{V}(\Sigma)$ at $X_{0}$.

Proof Use the same Liapunov-Schmidt reduction technique as in the preceding section, and observe that the reduced map $g$ is $\Gamma \times \mathbf{S}^{1}$-equivariant, where the $\mathbf{S}^{\mathbf{1}}$-action induced by phase shift is identical to the $\mathbf{S}^{\mathbf{1}}$-action induced by $A_{0}$. Equivariance implies that $g: F_{V}(\Sigma) \times \mathbb{R} \rightarrow \operatorname{Fix}_{V}(\Sigma)$ has the form (3.3). Using (E3a) we can identify $\operatorname{Fix}_{V}(\Sigma)$ with $\mathbb{C}$. Now proceed as in the proof of Theorem 3.1 .

Remark 4.3 It is also possible to prove this equivariant version of the Liapunov center theorem by using the trick of adding dissipation to the Hopf theorem; see Golubitsky, Stewart and Schaeffer [1988], Exercise 4.4, p. 283. 


\section{Remarks and generalizations}

(a) The entire machinery of finding two-dimensional fixed-point subspaces in $\Gamma$ equivariant Hopf bifurcation can now be applied to finding periodic solutions in systems with first integrals. For example, in $\mathbf{O}(2)$-equivariant systems with $\operatorname{dim} V=4$, Theorem 4.2 produces both rotating and standing waves.

(b) As noted in Golubitsky and Stewart [1993], there are surprising grouptheoretic conditions restricting those $\Gamma$ that can produce two-dimensional fixed-point subspaces. Let $\pi_{\Gamma}: \Gamma \times \mathbf{S}^{1} \rightarrow \Gamma$ be the projection and let $H=\pi_{\Gamma}(\Sigma)$. Note that $H$ is isomorphic to $\Sigma$. Let $K=(\Gamma \times\{0\}) \cap \Sigma$. Indeed, we can think of $\Sigma$ as a twisted subgroup of $\Gamma \times S^{1}$; that is, uniqueness of solutions guarantees the existence of a group homomorphism $\theta: H \rightarrow \mathrm{S}^{1}$ such that $(\gamma, \theta(\gamma)) \in \Sigma$ for all $\gamma \in H$. Note that $K=\operatorname{ker} \theta$, from which it follows that $K$ is normal in $H$ and $H / K$ is isomorphic to a Lie subgroup of $\mathbf{S}^{\mathbf{1}}$ and hence is either cyclic or $\mathbf{S}^{\mathbf{1}}$. In any case $H / K$ is abelian. The following theorem is proved in Golubitsky and Stewart [1993]: If there is any representation $V$ in which $\Sigma$ has a two-dimensional fixed-point subspace, then $H / K$ is a maximal abelian subgroup of $N_{\Gamma}(K) / K$ where $N_{\Gamma}(K)$ is the normalizer of $K$ in $\Gamma$.

(c) In the case of a Hamiltonian system with symmetry group $G$, the method of the preceding section may be applied to the new Hamiltonian system obtained by symplectically reducing by the continuous part of a symplectic group action (namely, by the connected component of the identity of $G$ ) at a regular value of the momentum map. (See, for example, Marsden [1992] for the general set-up. The case of a singular value of the momentum map is very interesting and the above ideas would need to be modified to cover it. It is probable that a symplectic slice theorem will be useful for this situation.) After reduction by the continuous group, one is in general left with a discrete symmetry group, which can be used for the group $\Gamma$ in the above result. The application of the Liapunov center theorem to the reduced problem will produce relative equilibria, so that one will get relative periodic orbits (often tori) in the original space.

(d) Montaldi, Roberts and Stewart [1988] deduce Theorem 4.2 from an equivariant version of the Weinstein-Moser theorem - see their Remark 1.2(b). However, they do not give the proof of local smoothness of the manifold in any cletail.

(e) First integrals coupled with Liapunov-Schmidt reduction can be used to recover the periodic solutions found in the zero eigenvalue bifurcations studied in Golubitsky and Stewart [1987]. The advantage of the approach of the present paper is that these families of periodic solutions will exist even when the Hamiltonian has many degrees of freedom. A similar conclusion can be obtained using the splitting lemma.

(f) The existence of integrals in the Liapunov-Schmidt reduction works in finite differentiability since the proof depends only on the standard implicit function theorem. Proofs of results like the Liapunov center theorem in finite differentiability can be based on a finite differentiability version of (3.3), 
which we have not derived. (A related approach may be found in Vanderbauwhede [1990].) It is clear that if the original vector hield is of class $C^{2 k+1}$ then the invariant functions $p$ and $q$ will be only of class $C^{k}$. (See, for example, Abraham, Marsden and Ratiu [1988] Section 2.4 for this type of argument.) This information is sufficient to prove the existence of the manifold of periodic solutions in the Liapunov center theorem, but some care is needed to determine the exact degree of differentiability of that manifold.

(g) As mentioned, possession of a first integral is just one kind of extra structure that a system may have. Of course, symmetry provides extra structure, but this can be exploited directly: the symmetry of the system transfers to the bifurcation equation. In Remark (i) we show how to formulate the condition of being Hamiltonian as an implicit constraint. In the next section we show how, in some cases, this implicit constraint can be recast into the condition that $g$ is Hamiltonian and use this observation to lay the foundation for an approach to finding periodic solutions of Hamiltonian systems.

(h) The condition of being a gradient system can also be formulated as an implicit constraint. Suppose that we seek equilibria of a gradient system on $\mathbb{R}^{n}$ (or more generally on a Riemannian manifold), say

$$
\dot{x}=\nabla h(x) \text {. }
$$

We use the standard inner product on $\mathbb{R}^{n}$ denoted $\langle$,$\rangle , and use it to identify$ $\mathbb{R}^{\prime \prime}$ with its dual space. We seek zeros of the map $F(x)=\nabla h(x)$, assuming, as usual that $F(0)=0$. In this case, the linearized operator $L=d F(0)$ is self-adjoint, so the splitting in the domain and range space are identical, namely $\mathbb{R}^{n}=\operatorname{ker} L \oplus$ range $L$. Let $\mathcal{X}=\mathbb{R}^{n}, \mathcal{Y}=\mathbb{R}^{n}$ and $\mathcal{W}=\mathbb{R}$. Define the map

$$
\Phi: \mathcal{X} \times \mathcal{Y} \rightarrow \mathcal{W}
$$

by

$$
\Phi(x, y) \cdot u=\langle y, u\rangle-d h(x) \cdot u .
$$

The implicit constraint

$$
\Phi(x, F(x))=0
$$

holds. The condition imposed on the bifurcation equation is

$$
0=\varphi(k, g(k)) \cdot u=\langle g(k), u\rangle-d h(k+w(k)) \cdot u .
$$

However, by definition of the implicit function $w$, we have

$$
\begin{aligned}
d h(k+w(k)) \cdot(d u ;(k) \cdot u) & =\langle\nabla h(k+w(k)),(d w(k) \cdot u)\rangle \\
& =\langle\nabla h(k+w(k)), \mathbf{P}(d w(k) \cdot u)\rangle \\
& =\langle\mathbf{P} \nabla h(k+w(k)),(d w(k) \cdot u)\rangle \\
& =0 .
\end{aligned}
$$

Therefore, the implicit constraint can be written as

$$
0=\varphi(k, g(k)) \cdot u=\langle g(k), u\rangle-d h(k+w(k)) \cdot(u+d w(k) \cdot u) .
$$

By the chain rule, we can rewrite this as

$$
0=\langle g(k), u\rangle-d \bar{h}(k) \cdot u
$$


where $\bar{h}(k)=h(k+w(k))$, so that $g$ is a gradient field, which recovers a result of Rabinowitz [1977].

(i) We can modify the above example to find equilibria of a Hamiltonian system, by replacing the inner product by the symplectic structure. By definition a Hamiltonian vector field $X_{H}$ on a symplectic vector space $(V, \Omega)$ satisfies

$$
\Omega\left(X_{H}(z), u\right)=d H(z) \cdot u \text {. }
$$

Let $F(z)=X_{H}(z)$, and choose $\mathcal{X}=V, \mathcal{Y}=V$ and $\mathcal{W}=V^{*}$, the dual space. Define the map

$$
\Phi: \mathcal{X} \times \mathcal{Y} \rightarrow \mathcal{W}
$$

by

$$
\Phi(z, y) \cdot u=\Omega(y, u)-d H(z) \cdot u .
$$

Again the bifurcation equation inherits an implicit constraint reflecting the Hamiltonian condition. In fact, as we shall see in the next section, under appropriate hypotheses that play the role of the self-adjoint ness in the preceding example, the map $g$ will be a Hamiltonian vector field. This way of phrasing the implicit constraint will play an important role in what follows.

\section{The Hamiltonian constraint}

We begin this section by amplifying the remark at the end of the last section and then we consider the problem of the existence of periodic orbits for Hamiltonian systems (possibly with symmetry) near equilibria.

6.1 Conditions for the bifurcation map to be Hamiltonian Let $V$ be a symplectic vector space (possibly infinite-dimensional) with symplectic form $\Omega$ and let $F: V \times \Lambda \rightarrow V$ be a Hamiltonian vector field with Hamiltonian $H: V \times \Lambda \rightarrow \mathbb{R}$. Here, $\Lambda$ denotes a space of parameters that we will, as usual, suppress for the moment.

Assume, as in the general Liapunov-Schmidt procedure, that $F(0)=0$. Let $L: V \rightarrow V$ be the linearization of $F$ at the origin. Recall that the generalized kernel of $L$ is a symplectic subspace of $V$. (See Golubitsky and Stewart [1987], for example.) As is well known, and is easy to check, the operator $L$ is Hamiltonian with Hamiltonian the quadratic form associated with $d^{2} H(0)$, the second derivative of $H$ at the origin. It follows that $L^{*}$ is also Hamiltonian with Hamiltonian the quadratic form associated to $J d^{2} H(0) J^{*}$. Another way to express the fact that $L$ is Hamiltonian is to say that $L$ is $\Omega$-skew: $\Omega(L u, v)=-\Omega(u, L v)$ for all $u, v \in V$.

Assume that

$$
\Omega(u, v)=\langle\langle J u, v\rangle\rangle
$$

where $J: V \rightarrow V$ is a complex structure and where $\langle\langle\rangle$,$\rangle is a real inner product on$ $V$. This is the standard relation between complex and symplectic structures (see, for example, Abraham and Marsden [1978].) In these terms, the skew symmetry of $L$ reads

$$
\langle\langle J L u, v\rangle\rangle=-\langle\langle J u, L v\rangle\rangle
$$

A check of the definitions shows that $J(\operatorname{ker} L)=\operatorname{ker} L^{*}$. That is, the complex structure maps the kernel of $L$ to that of $L^{*}$. To show this, let $u \in \operatorname{ker} L$. To show that $J u \in \operatorname{ker} L^{*}$ we write, for any $v \in V$,

$$
\left\langle\left\langle L^{*} J u, v\right\rangle\right\rangle=\langle\langle J u, L v\rangle\rangle=-\langle\langle J L u, v\rangle\rangle=0 .
$$


Thus, $L^{*} J u=0$, as we claimed.

Consistent with the notation in the general Liapunov-Schmidt procedure, let $\mathcal{K}=\operatorname{ker} L, \mathcal{N}=\operatorname{ker} L^{*}, \mathcal{R}=\operatorname{range} L$ and $\mathcal{M}=$ range $L^{*}$. A main hypotheses that will be appropriate for the Liapunov center theorem and resonance bifurcations is as follows:

(S) The complex structure $J$ leaves $\operatorname{ker} L$ invariant: $\operatorname{ker} L=\operatorname{ker} L^{*}$.

The formula relating $\Omega$ and $J$ shows that if a subspace $W \subset V$ is invariant under $J$, then it is automatically symplectic. Conversely, if it is symplectic, then writing $V=W \oplus W^{\Omega}$ where $W^{\Omega}$ is the $\Omega$ orthogonal complement of $W$, and choosing an associated inner product and complex structure on each summand, one sees that it is possible to choose the inner product and complex structure on $V$ so that $W$ is invariant under $J$. Because of this remark, we see that the above assumption is automatic if the kernel of $L$ equals the generalized kernel (and, if necessary, the inner product and the complex structure are adjusted). In this sense, the assumption is akin to a semisimplicity assumption.

Proposition 6.1 Under hypothesis (S), the spaces $\mathcal{R}$ and $\mathcal{N}$ as well as the spaces $\mathcal{K}$ and $\mathcal{M}$ are $\Omega$ orthogonal complements.

Proof By (S), the kernels of $L$ and $L^{*}$ are identical. It follows that the ranges are identical as well. If $v=L u$ and if $L y=0$, then

$$
\Omega(v, y)=\langle\langle J v, y\rangle\rangle=-\langle\langle L u, J y\rangle\rangle=\left\langle\left\langle u, L^{*} J y\right\rangle\right\rangle=0
$$

since, by nypothesis, $J y$ is in the kernel of $L$, that is, in the kernel of $L^{*}$. The proposition now follows.

Hypothesis (S) implies that the mapping $g$ maps $\mathcal{K} \rightarrow \mathcal{K}$. With the above assumption, it therefore makes sense to think of $g$ as a vector field on the symplectic vector space $\mathcal{K}$. We now make a simple but crucial observation:

Theorem 6.2 Under assumption (S), the map $g$ is a Hamiltonian vector field with Hamiltonian $h(k)=H(k+w(k))$. Moreover, $g$ and the function $h$ have the same invariance properties as the given Hamiltonian $H$.

Proof Let $k_{1} \in \mathcal{K}$ and recall that $g(k)=(I-P) F(k+w(k))$. Use the fact that $D w(k) \cdot k_{1} \in \mathcal{M}$ and the fact that $P F(k+w(k))=0$ is the defining property of the implicit function $w$ and Proposition 6.1 to verify

$$
\begin{aligned}
\Omega\left(g(k), k_{1}\right) & =\Omega\left(g(k), d w(k) \cdot k_{1}+k_{1}\right) \\
& =\Omega\left(F(k+w(k)), d w(k) \cdot k_{1}+k_{1}\right) .
\end{aligned}
$$

Since $F$ is Hamiltonian, we can use the chain rule to write the last expression as

$$
d H(k+w(k)) \cdot\left(d w(k) \cdot k_{1}+k_{1}\right)=d h(k) \cdot k_{1} .
$$

Thus, $g$ is a Hamiltonian vector field with Hamiltonian function $h$. Invariance properties follow in the usual way from uniqueness, assuming that the group action preserves the symplectic form and the complex structure.

In particular, in this case we have reformulated the problem to one of searching for equilibria of a Hamiltonian vector field defined on the kernel. In what follows, we 
will show how to also use this result for finding periodic orbits by casting the problem into one of finding the equilibria of an infinite-dimensional Hamiltonian system on loop space. See Vanderbauwhede and van der Meer [1994] for an alternative discussion of the Hamiltonian structure of the bifurcation equations.

6.2 Periodic orbits and the loop space We start with a Hamiltonian system $\dot{z}=X_{H}(z)$ on a symplectic vector space $(E, \omega)$. As we have noted before, eventually $H$ will also depend on a bifurcation parameter. (We will be assuming that the space $E$ is finite-dimensional for simplicity, but in principle the methods that we outline here will apply to infinite-dimensional problems too; however, as is known, the resonance assumptions that are necessary in the infinite-dimensional case are severe using these methods.) We will be applying the above theorem not to this Hamiltonian system, but to an associated Hamiltonian system on loop space, to be described below. The point of view of using the loop space was used by Weinstein in one approach to the Weinstein-Moser theorem (see Weinstein [1978]) and it is also related to the method used by Bridges [1990].

We may assume that $E=\mathbb{C}^{n}$ and that $\omega$ is in canonical form, that is, $\omega$ is the skew-symmetric bilinear form given by

$$
\omega\left(z_{1}, z_{2}\right)=-2 \operatorname{Im}\left\langle z_{1}, z_{2}\right\rangle \text {. }
$$

The factor of -2 is of course conventional and corresponds to the standard choice of symplectic structure. Also, the inner product here stands for the standard Her. mitian inner product.

Introduce the loop space $\mathcal{C}_{2 \pi}^{1}$ of $C^{1}$ maps $\mathbf{S}^{1} \rightarrow E$ and let $\mathcal{C}_{2 \pi}^{0}$ be the corresponding space of $C^{0}$ maps. As in Section 3, define

$$
F: \mathcal{C}_{2 \pi}^{1} \times \mathbb{R} \rightarrow \mathcal{C}_{2 \pi}^{0}
$$

by

$$
F(u, \tau)=(1+\tau) \frac{d u}{d s}-f(u),
$$

where $f=X_{\|}$. The Liapunov-Schmidt procedure is now applied to the map $F$.

Since the vector field $f=X_{H}$ is Hamiltonian, the identity

$$
\omega\left(X_{H}(u), v\right)=d H(u) \cdot v
$$

holds for all $v \in \mathbb{R}^{2 n}$. Define the map

$$
\Phi: \mathcal{C}_{2 \pi}^{1} \times \mathbb{R} \times \mathcal{C}_{2 \pi}^{0} \rightarrow\left[\mathcal{C}_{2 \pi}^{1}\right]^{*}
$$

by

$$
\Phi(u, \tau, v) \cdot U=\int_{0}^{2 \pi}\left\{\omega\left(v-(1+\tau) \frac{d u}{d s}, U\right)+d H(u) \cdot U\right\} d s
$$

The implicit constraint

$$
\Phi(u, \tau, F(u . \tau))=0
$$

is a restatement of the condition that the vector field $f$ is Hamiltonian with Hamiltonian function $H$. This condition can also be rephrased as saying that the map 
$F$, regarded as a (parameter-dependent) vector field on $\mathcal{C}_{2 \pi}^{1}$, is Hamiltonian with respect to the weak symplectic form

$$
\Omega(u, v)=\frac{1}{2 \pi} \int_{0}^{2 \pi} \omega(u(s), v(s)) d s,
$$

and with the Hamiltonian function

$$
\mathcal{H}(u, \tau)=\frac{1}{2 \pi} \int_{0}^{2 \pi}\left\{\frac{1}{2} \omega\left((1+\tau) \frac{d u}{d s}, u\right)-H(u)\right\} d s
$$

We can also put a real inner product and a complex structure on loop space by averaging the corresponding objects on $E$ around loops, as we did with the symplectic structure.

In this approach, we take the symplectic vector space to which the general theory is to be applied to be the loop space $V=\mathcal{C}_{2 \pi}^{0}$. Of course, there is the usual derivative loss, so the vector field $F$ is really only defined on the dense subspace $\mathcal{C}_{2 \pi}^{1}$. Moreover, the real inner product with respect to which adjoints are taken is an $L^{2}$ inner product and so $V$ is not complete. In addition, the symplectic form is only a weak symplectic form. However, as in Weinstein [1978] and Chernoff and Marsden [1974], while these are important technicalities, they are easily taken care of using ellipticity; they are similar to the technicalities that occur in this approach to the Hopf bifurcation (as we mentioned before, the reader can consult Golubitsky and Schaeffer [1985], p. 332 for additional information).

Theorem 6.2 shows that if the kernel of the linearized equations is symplectic and arranged to be invariant under the complex structure, then the bifurcation equation will be a Hamiltonian vector field. To be able to apply this, we need to do two things. First, we need to calculate the linearized equations and second, we need to calculate the induced symplectic structure on the kernel of the linearization and check that it is nondegenerate. In the remainder of this section we shall show how to do this for the Liapunov center theorem and later we shall show how to implement it for the case of resonance bifurcations.

6.3 The Hamiltonian Liapunov center theorem Now we apply the Hamiltonian version of the Liapunov-Schmidt procedure developed above to the case of the Hamiltonian Liapunov center theorem. As in (3.2) the linearization

$$
L: \mathcal{C}_{2 \pi}^{1} \rightarrow \mathcal{C}_{2 \pi}^{0}
$$

is

$$
L v=\frac{d v}{d s}-A_{0} v
$$

where $A_{0}=d f(0)$. Since the linear map $L$ is Fredholm of index zero we may use the $L^{2}$-orthogonal splittings

$$
\begin{aligned}
& \mathcal{C}_{2 \pi}^{1}=\mathcal{K} \oplus \text { range } L^{*} \\
& \mathcal{C}_{2 \pi}^{0}=\text { range } L \oplus \mathcal{N}
\end{aligned}
$$

where $\mathcal{K}=\operatorname{ker} L$ and $\mathcal{N}=\operatorname{ker} L^{*}$. The Liapunov-Schmidt reduced map obtained from $F$ is

$$
g: \mathcal{K} \times \mathbb{R} \rightarrow \mathcal{N}
$$

Next, we compute the kernel of $L$ under the given eigenvalue assumptions. First of all, note that the real eigenspace of $A_{0}$ corresponding to eigenvalues $\pm i$ (with 
the frequency set equal to one, as before) is of real dimension two and is coincident with the generalized eigenspace since the eigenvalues are assumed to be simple. Thus, this space is symplectic. (As before, see, for example, Golubitsky and Stewart [1987] for a discussion of this Williamson type of result.) We can choose the complex structure on $E$ so that this eigenspace is the one complex dimensional space corresponding to the eigenvalue $i$ and that the operator $A_{0}$ is given by multiplication by $i$ on this space. We can also assume that the other eigenspaces are $\omega$-orthogonal to it. We can now realize $E$ as $\mathbb{C}^{n}$ with this eigenspace given by the first factor. Thus, in this complex representation, the complex eigenspace with eigenvalue $i$ (i.e., the two-dimensional real eigenspace corresponding to eigenvalues $\pm i)$ is given by the first component. Let $v_{0}=(1,0, \ldots, 0)$ denote this eigenvector.

The kernel of $L$ was given previously in real terms, but in complex terms, it is simply the two-dimensional subspace of the loop space given by the set of all loops of the form

$$
v(s)=e^{i s} z v_{0}
$$

for arbitrary complex $z$. This space is clearly invariant under the complex structure on loop space that was described earlier. Also, we see explicitly that this space is symplectic by noting that its symplectic structure is given, using self explanatory notation, by

$$
\begin{aligned}
\Omega\left(v_{1}, v_{2}\right) & =\frac{1}{2 \pi} \int_{0}^{2 \pi} \omega\left(e^{i s} z_{1} v_{0}, e^{i s} z_{2} v_{0}\right) \\
& =\frac{1}{2 \pi} \int_{0}^{2 \pi}-2 \operatorname{Im}\left\langle e^{i s} z_{1} v_{0}, e^{i s} z_{2} v_{0}\right\rangle \\
& =-2 \operatorname{Im} z_{1} \bar{z}_{2} .
\end{aligned}
$$

In other words, the symplectic structure on the kernel of $L$, after identification with $\mathbb{C}$, is the standard one. Thus, the general theory applies to show that the map $g$ that occurs in the bifurcation equation $g=0$ is indeed a Hamiltonian vector field.

Recall from (3.3) that due to $\mathbf{S}^{1}$-equivariance, the bifurcation map has the form

$$
g: \mathbb{C} \times \mathbb{R} \rightarrow \mathbb{C}
$$

where

$$
g(z, \tau)=p z+q i z
$$

and $p$ and $q$ are real valued functions of $|z|^{2}$ and $\tau$. In Section 3 we used $(3.4)$ to show that $p$ vanishes whenever $q$ vanishes. However, in the present case, knowing that $g$ is Hamiltonian implies that it has the form

$$
g(z)=-2 i \frac{\partial H}{\partial \bar{z}}
$$

for a real valued function $H$ of $(z, \bar{z})$. In particular, $g$ is purely imaginary, and so we have the identity $p=0$. 
6.4 Conditions imposed on the bifurcation equation In this subsection we consider the situation when the hypothesis (S) might fail for the linearization. This will be needed in the next section when we search for periodic orbits in the Hamiltonian Hopf bifurcation. In this case, we still have an implicit constraint as is guaranteed by the general theory described earlier. To do this, we return to the implicit constraint on loop space, condition (6.3), but we do not necessarily assume hypothesis $(\mathrm{S})$.

As in (3.2) the linearization

$$
L: \mathcal{C}_{2 \pi}^{1} \rightarrow \mathcal{C}_{2 \pi}^{0}
$$

is

$$
L v=\frac{d v}{d s}-A_{0} v,
$$

where $A_{0}=d f(0)$. Since the linear map $L$ is Fredholm of index zero (we showed above that in general the kernel and the cokernel of $L$ are isomorphic, and so have the same dimension), we may use the splittings

$$
\begin{aligned}
& \mathcal{C}_{2 \pi}^{!}=\mathcal{K} \oplus \text { range } L^{*} \\
& \mathcal{C}_{2 \pi}^{0}=\text { range } L \oplus \mathcal{N}
\end{aligned}
$$

where $\mathcal{K}=\operatorname{ker} L$ and $\mathcal{N}=\operatorname{ker} L^{*}$. The Liapunov-Schmidt reduced map obtained from $F$ is

$$
g: \mathcal{K} \times \mathbb{R} \rightarrow \mathcal{N}
$$

We use (6.3) to determine a condition that is independent of the implicit function $w$ used to define $g$. For this purpose we choose the function $U$ in (6.3) to be $d u / d s$. With this choice it is clear that the last term in (6.3) vanishes, since it is the $s$-derivative of $H(u(s))$ and $u$ is periodic. It is also obvious that the term

$$
\omega((1+\tau) d u / d s, d u / d s)
$$

is zero. Thus,

$$
\Psi(u, \tau, v)=\int_{0}^{2 \pi} \omega\left(\frac{d u}{d s}, v\right) d s
$$

is an implicit constraint for $F$. Setting $u=k+w(k, \tau)$ we obtain the identity

$$
\psi(k, \tau) \equiv \int_{0}^{2 \pi} \omega\left(\frac{d k}{d s}+\frac{d w}{d s}, g(k, \tau)\right)=0
$$

from the induced implicit constraint on $g$.

Lemma 6.3 We have

$$
\int_{0}^{2 \pi} \omega\left(L^{*} v, V\right) d s=-\int_{0}^{2 \pi} \omega\left(v, L^{*} V\right) d s .
$$

for all $v, V \in \mathcal{C}_{2 \pi}^{1}$.

Proof Recall that

$$
L \cdot V=-\frac{d V}{d s}-A_{0}^{*} V
$$


Since $v$ and $V$ are periodic, integration by parts yields

$$
\begin{aligned}
\int_{0}^{2 \pi} \omega\left(v, L^{*} V\right) d s & =\int_{0}^{2 \pi}\left\{\omega\left(v,-\frac{d V}{d s}-A_{0}^{*} V\right)\right\} d s \\
& =\int_{0}^{2 \pi}\left\{\omega\left(\frac{d v}{d s}, V\right)+\omega\left(v,-A_{0}^{*} V\right)\right\} d s
\end{aligned}
$$

Earlier we showed that both $A_{0}$ and $A_{0}^{*}$ are Hamiltonian vector fields. This implies in particular that:

$$
\omega\left(v, A_{0}^{*} V\right)=-\omega\left(A_{0}^{*} v, V\right)
$$

This shows that

$$
\begin{aligned}
\int_{0}^{2 \pi}\left\{\omega\left(\frac{d v}{d s}, V\right)+\omega\left(v,-A_{0}^{*} V\right)\right\} d s & =\int_{0}^{2 \pi} \omega\left(\frac{d v}{d s}+A_{0}^{*} v, V\right) d s \\
& =-\int_{0}^{2 \pi} \omega\left(L^{*} v, V\right) d s
\end{aligned}
$$

as desired.

Since $d w / d s \in$ range $L^{*}$ and $g \in \operatorname{ker} L^{*}$, this lemma establishes the symplectic orthogonality condition

$$
\int_{0}^{2 \pi} \omega\left(\frac{d w}{d s}, g(k, \tau)\right)=0
$$

This proves the following:

Theorem 6.4 The bifurcation mapping $g$ satisfies the implicit constraint

$$
\psi(k, \tau)=\int_{0}^{2 \pi} \omega\left(\frac{d k}{d s}, g(k, \tau)\right)=0 .
$$

\section{Part II Hamiltonian-Hopf Bifurcations}

\section{Introduction}

A Hamiltonian-Hopf bifurcation occurs in a Hamiltonian system of ODEs when, as a parameter $\lambda$ in the Hamiltonian is varied, two pairs of simple purely imaginary eigenvalues of the Jacobian at an equilibrium collide and form a quadruplet of complex eigenvalues. The splitting of eigenvalues into the complex plane forces the critical purely imaginary eigenvalues to be geometrically simple (though algebraically double). The Hamiltonian Hopf bifurcation has a long history that we shall not attempt to survey here. We refer to Meyer and Schmidt [1971], Abraham and Marsden [1978] and Arnol'd [1988] for discussions and some references. We discuss some of the relevant literature in the introduction to Part III.

Van der Meer [1985] studied this bifurcation and, through the application of normal form theory and singularity theory developed for the purpose, was able to classify the periodic solutions that are spawned by this resonance. Bridges [1990] showed that the periodic solutions in a Hamiltonian-Hopf bifurcation can be obtained using $\mathbb{Z}_{2}$ singularity theory with a distinguished parameter as developed in Golubitsky and Langford [1981]. In this Part we show that Bridges's results can be recovered as an example of the use of implicit constraints. We summarize the advantages of this approach. 
- No discussion of the effects of the tails of Taylor series - such as occurs in all normal form analyses - need be given.

- The proof works equally easily for $2 n$-dimensional systems as for four-dimensional systems. This fact may allow eventually the derivation of a formula describing the alternatives in the Hamiltonian-Hopf bifurcation such as now exists for Hopf bifurcation.

The disadvantages of this approach are summarized by:

- The issue of the linearized stability of the periodic solutions is not addressed (though a strengthening of the reduction technique such as occurs in the analysis of Hopf bifurcation, see Golubitsky, Stewart and Schaeffer [1988] Chapter XVI Section 6, might be able to recover this information).

- Dynamics other than periodic solutions - such as the existence of invariant tori - are not discussed. Again, we feel that this can be achieved with additional effort.

After rescaling we can assume that the critical eigenvalues are at $\pm i$. We use the Liapunov-Schmidt procedure for finding periodic solutions that are approximately $2 \pi$-periodic. As we will show, the assumption that the eigenvalues are geometrically simple permits a reduction of the problem of finding $2 \pi$-periodic solutions to one of solving an equation of the form

$$
g(z, \lambda, \tau)=0
$$

where

$$
g: \mathbb{C} \times \mathbb{R} \times \mathbb{R} \rightarrow \mathbb{C} .
$$

As in the proof of the Liapunov center theorem, $S^{1}$ phase-shift symmetry on $2 \pi$ periodic functions implies that $g$ is $\mathbf{S}^{1}$-equivariant. Equivariance in turn guarantees that

$$
g(x, \lambda, \tau)=p\left(x^{2}, \lambda, \tau\right) x+q\left(x^{2}, \lambda, \tau\right) i x,
$$

where $p$ and $q$ are real-valued functions. Solving $g=0$ for nonzero solutions reduces to solving the pair of equations $p=q=0$. As in Bridges [1990] we will show that the $\mathbf{S}^{1}$-invariant function $q$ vanishes identically. (Note the difference with the Liapunov center theorem where $p$ vanishes identically.) Thus the problem of finding periodic solutions in the original system of ODEs reduces, near a point where a HamiltonianHopf bifurcation occurs, to finding solutions of

$$
p\left(x^{2}, \lambda, \tau\right) x=0 .
$$

As van der Meer notes, the solutions to (7.1) are most easily found by swapping the roles of $\lambda$ and $\tau$; that is, think of the perturbed period parameter $\tau$ as the bifurcation parameter and the system parameter $\lambda$ as the unfolding parameter. The main technical result of this Part is the verification of the following:

Theorem 7.1

(a) $p(0,0,0)=0, \quad p_{\tau}(0,0,0)=0, \quad p_{\tau \tau}(0,0,0)>0$.

(b) Generically $p_{\lambda}(0,0,0)$ and $p_{x^{2}}(0,0,0)$ are nonzero. 
$\lambda<0$

$\lambda=0$

$\lambda<0$

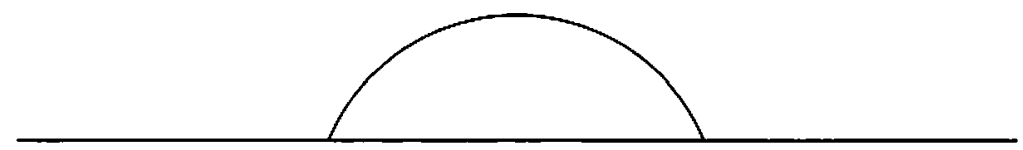

Figure $1\left(x^{2}+\tau^{2}+\lambda\right) x=0$.

Theorem 7.1 shows that solving (7.1) is equivalent to solving the (singularity theory) normal form equation:

$$
\left( \pm x^{2} \pm \lambda+\tau^{2}\right) x=0 .
$$

The bifurcation diagrams associated with these solutions are given in Figures 1 and 2. Note that when $\lambda<0-$ which corresponds to the eigenvalues of the Jacobian being purely imaginary - there are two branches of periodic solutions emanating from the trivial equilibrium. These solutions are just the ones guaranteed by the Liapunov center theorem.

We end this introduction by discussing the structure of Part II. In the next section we discuss the linear algebra associated with a Hamiltonian-Hopf bifurcation. In Section 9 we introduce the generalities of the Liapunov-Schmidt reduction while in Section 10 we show how the Hamiltonian implicit constraint allow us to conclude that the function $q$ is a multiple of $p$ and that it even has to vanish identically. In Section 11 we prove Theorem 7.1 and in the last section we use singularity theory to derive (7.2).

\section{The general setting}

Let $H: \mathbb{R}^{2 n} \times \mathbb{R} \rightarrow \mathbb{R}$ be a Hamiltonian parametrized by a system parameter $\lambda$, and let

$$
\frac{d X}{d s}=f(X, \lambda)
$$

be the corresponding Hamiltonian system of ODEs. We assume that at $\lambda=\lambda_{0}$ the svstem (8.1) undergoes a Hamiltonian-Hopf bifurcation at the equilibrium $X=X_{0}$. We simplify notation by setting $X_{0}=0$ and $\lambda_{0}=0$. Thus

$$
f(0,0)=d H(0,0)=0 .
$$


$\lambda>0$

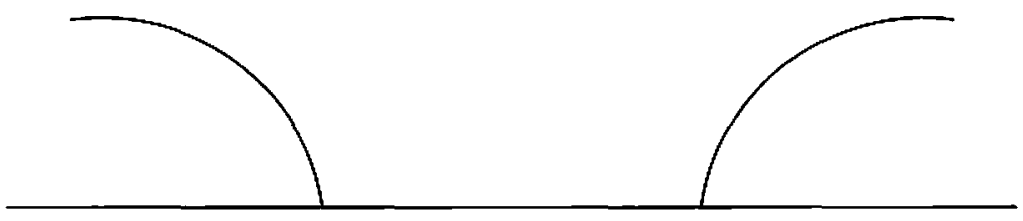

$\lambda=0$

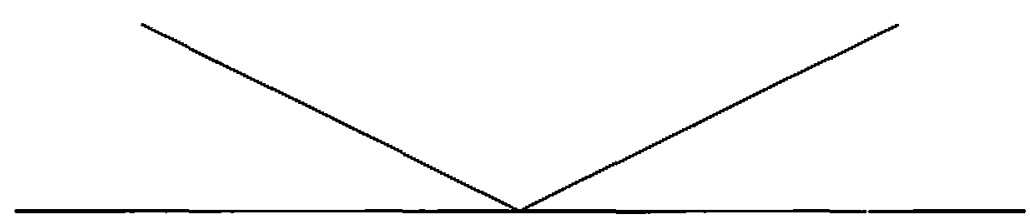

$\lambda<0$

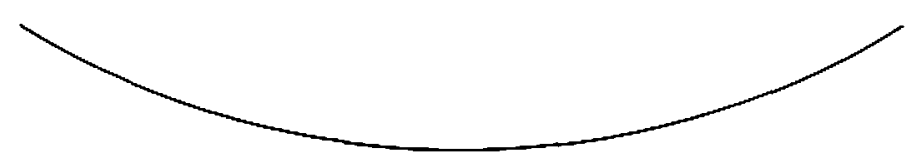

Figure $2\left(-x^{2}+\tau^{2}+\lambda\right) x=0$.

In a Hamiltonian-Hopf bifurcation two pairs of simple purely imaginary eigenvalues collide on the imaginary axis as $\lambda$ is varied. Generically, after the collision, the critical eigenvalues form a quadruplet of eigenvalues in the complex plane. Indeed, generically at criticality, the generalized eigenspace $V$ corresponding to the critical eigenvalues is a four-dimensional subspace of $\mathbb{R}^{2 n}$ on which

$$
A_{0}=d f(0,0)
$$

is nilpotent and has double purely imaginary eigenvalues $\pm i w$. We rescale time in (8.1) and assume that

$$
\omega_{0}=1 \text {. }
$$

Nilpotency implies that $A_{0}$ has a two-dimensional invariant subspace

$$
V_{0}=\operatorname{ker}\left(A_{0}^{2}+I\right) \text {, }
$$

and a complementary two-dimensional subspace $V_{1}$ mapped isomorphically onto $V_{0}$ by $A_{0}^{2}+I$.

The linear map $A_{0} \mid V$ has a normal form that can be described by choosing an appropriate basis, as follows. Choose $v_{1}, v_{2}$ such that

$$
V_{0}=\mathbb{R}\left\{v_{1}, v_{2}\right\}
$$

and

$$
A_{0}\left(v_{1}\right)=v_{2} \quad \text { and } \quad A_{0}\left(v_{2}\right)=-v_{1}
$$

Next, choose a basis

$$
V_{1}=\left\{v_{3}, v_{1}\right\}
$$


where

$$
A_{0}\left(v_{3}\right)=v_{1}+v_{4} \quad \text { and } \quad A_{0}\left(v_{4}\right)=v_{2}-v_{3} .
$$

In this basis, the matrix form of $A_{0} \mid V$ is

$$
A_{0} \mid V=\left(\begin{array}{ll}
J & I \\
0 & J
\end{array}\right)
$$

where

$$
J=\left(\begin{array}{cc}
0 & -1 \\
1 & 0
\end{array}\right)
$$

At a generic Hamiltonian-Hopf bifurcation we assume nonresonance of the critical eigenvalues; that is, we assume that $k i$ is not an eigenvalue of $d f(0,0)$ when $k=0, \pm 2, \pm 3, \ldots$. Since 0 is not an eigenvalue, the implicit function theorem implies that there is a smooth branch of equilibria for (8.1); that is,

$$
f\left(u_{0}(\lambda), \lambda\right)=0 \text {, }
$$

where $u_{0}(0)=0$. We may change coordinates in $u$ so that this family of equilibria is at 0 ; that is, $u_{0}(\lambda) \equiv 0$. Thus, we may assume

$$
f(0, \lambda)=0 \text { for all } \lambda \text {. }
$$

\section{The reduced system}

In the present context the operator $F$ in (3.1) depends on the parameters $\tau$ and $\lambda$, and we define $F: \mathcal{C}_{2 \pi}^{1} \times \mathbb{R} \times \mathbb{R} \rightarrow \mathcal{C}_{2 \pi}^{0}$ by

$$
F(u, \lambda, \tau)=(1+\tau) \frac{d u}{d s}-f(u, \lambda) .
$$

As above, let $L$ be the linearization of $F$ at 0 ,

$$
L v=\frac{d v}{d s}-A_{0} v, \quad A_{0}=d f(0,0),
$$

and let $L^{*}$ be the adjoint operator

$$
L^{*} v=-\frac{d v}{d s}-A_{0}^{*} v .
$$

We view $A_{0}$ as an $n \times n$ matrix acting on $\mathbb{C}^{2 n}$. Then we can choose complex eigenvectors $v_{0}, v_{0}^{*} \in \mathbb{C}^{2 n}$ such that

$$
A_{0} v_{0}=-i v_{0} \quad \text { and } \quad A_{0}^{*} v_{0}^{*}=i v_{0}^{*}
$$

by setting

$$
v_{0}=v_{1}+i v_{2} \text { and } v_{0}^{*}=v_{3}+i v_{4} .
$$

Let $\cdot$ denote the ordinary inner product on $\mathbb{C}^{2 n}$. Then these choices satisfy

$$
v_{0}^{*} \cdot \overline{v_{0}}=v_{0}^{*} \cdot v_{0}=0,
$$

as a direct calculation using (9.1) shows.

Again we have the splittings (see Section 6)

$$
\begin{aligned}
& \mathcal{C}_{2 \pi}^{1}=\mathcal{K} \oplus \text { range } L^{*} \\
& \mathcal{C}_{2 \pi}^{0}=\text { range } L \oplus \mathcal{N}
\end{aligned}
$$


where $\mathcal{K}=\operatorname{ker} L$ and $\mathcal{N}=\operatorname{ker} L^{*}$. (Unlike in Hopf bifurcation, nilpotency of $A_{0}$ implies that we cannot choose $\mathcal{N}=\mathcal{K}$.) We compute

$$
\begin{aligned}
\mathcal{K} & =\left\{\operatorname{Re}\left(z e^{-i s} v_{0}\right) \mid z \in \mathbb{C}\right\} \\
\mathcal{N} & =\left\{\operatorname{Re}\left(z e^{-i s} v_{0}^{*}\right) \mid z \in \mathbb{C}\right\}
\end{aligned}
$$

In this way we have identified both $\mathcal{K}$ and $\mathcal{N}$ with $\mathbb{C}$, viewed as a two-dimensional real vector space. We denote elements of $\mathcal{K}$ by $v$ and elements of $\mathcal{N}$ by $v^{*}$. Finally, let

$$
E: \mathcal{C}_{2 \pi} \rightarrow \text { range } L
$$

be the projection onto range $L$ with kernel $\mathcal{N}$.

By the standard technique (implicit function theorem and $S^{1}$-equivariance) we can reduce the problem of finding zeros of $F$ to the solution of

$$
g(x, \lambda, \tau)=p\left(x^{2}, \lambda, \tau\right) x+q\left(x^{2}, \lambda, \tau\right) i x=0,
$$

where $x \in \mathbb{R}$. Here we can identify $x$ with the $2 \pi$-periodic function

$$
\hat{x}(s)=x \operatorname{Re}\left(e^{-i s} v_{0}\right) \text {. }
$$

\section{Dependence of $p$ and $q$}

In the case of the Liapunov center theorem we have seen that the function $p$ vanishes identically, and hence the solutions of the reduced system are determined by the equation $q=0$ (see Sections 3 and 6 ). As we will show in this section, in the case of the Hamiltonian Hopf bifurcation $q$ vanishes identically, and the structure of the set of periodic solutions is defined by $p=0$.

We proceed in a way analogous to our treatment of the Liapunov center theorem. First we use the implicit constraint induced by $\Phi$ in (3.4) to show that $q$ is a multiple of $p$. Then - using the implicit constraint of Section 6 - we prove that $q$ vanishes identically.

Define

$$
\Phi: \mathcal{C}_{2 \pi}^{1} \times \mathcal{C}_{2 \pi}^{0} \rightarrow \mathbb{R}
$$

by

$$
\Phi(u, v)=\int_{0}^{2 \pi} d H(u(s)) \cdot v(s) d s
$$

As in Section 3, but with parameters suppressed,

$$
\Phi(u, F(u)) \equiv 0 .
$$

This implies that

$$
\varphi: \mathcal{K} \times \mathcal{N} \rightarrow \mathbb{R}
$$

defined by

$$
\varphi(u, v)=\Phi(u+w(u), v)
$$

satisfies

$$
\varphi(u, g(u)) \equiv 0
$$

where $w$ is the implicitly defined function obtained from Liapunov-Schmidt reduction.

The general discussion in the previous paragraph implies that

$$
\int_{0}^{2 \pi} d H\left(u_{x}(s)\right) \cdot g(x, \lambda, \tau) d s=0
$$


where $u_{x}(s)$ is the element of $\mathcal{C}_{2 \pi}^{1}$ of the form $\hat{x}+O\left(x^{2}\right)$. Regarding $g(x, \lambda, \tau)$ as an element of $\mathcal{C}_{2 \pi}$ by

$$
z \mapsto \operatorname{Re}\left(z e^{-i s} v_{o}^{*}\right)
$$

and using the linearity of $\Phi$ and $\varphi$ in the second variable we obtain

$$
\begin{gathered}
p\left(x^{2}, \lambda, \tau\right) \int_{0}^{2 \pi} d H\left(u_{x}(s)\right) \cdot \operatorname{Re}\left(x e^{-i s} v_{0}^{*}\right) d s \\
+q\left(x^{2}, \lambda, \tau\right) \int_{0}^{2 \pi} d H\left(u_{x}(s)\right) \cdot \operatorname{Re}\left(i x e^{-i s} v_{0}^{*}\right) d s=0 .
\end{gathered}
$$

We want to show that there exists a function $r=r(x, \lambda, \tau)$ such that

$$
q\left(x^{2}, \lambda, \tau\right)=r(x, \lambda, \tau) p\left(x^{2}, \lambda, \tau\right)
$$

in a neighborhood of $x=0, x \neq 0$. For this to hold it is sufficient that the derivative of

$$
\Psi(x)=\int_{0}^{2 \pi} d H\left(u_{x}(s)\right) \cdot \operatorname{Re}\left(i e^{-i s} v_{0}^{*}\right) d s
$$

does not vanish at $x=0$. Since

$$
\operatorname{Re}\left(e^{-i s} v_{0}\right)=\cos (s) v_{1}+\sin (s) v_{2} \quad \text { and } \quad \operatorname{Re}\left(i e^{-i s} v_{0}^{*}\right)=\sin (s) v_{3}-\cos (s) v_{4}
$$

and

we find that

$$
d^{2} H(0,0)=\left(\begin{array}{rr}
0 & J \\
-J & I
\end{array}\right)
$$

$$
\begin{aligned}
\Psi^{\prime}(0) & =\int_{0}^{2 \pi} d^{2} H(0,0)\left(\operatorname{Re}\left(e^{-i s} v_{0}\right), \operatorname{Re}\left(i e^{-i s} v_{0}^{*}\right)\right) d s \\
& =2 \pi
\end{aligned}
$$

This proves (10.1).

We end this section by using the methods of Section 6 to show that $q \equiv 0$. First observe that all the considerations in that section leading to (6.8) are still valid if the operator $F$ additionally depends on a parameter. Hence, for elements $k \in \mathcal{K}=\operatorname{ker} L$, we have

$$
\psi(k, \lambda, \tau)=\int_{0}^{2 \pi} \omega\left(\frac{d k}{d s}, g(k, \lambda, \tau)\right)=0 .
$$

Substituting $g$ and using the $\mathbf{S}^{1}$-invariance of $p$ and $q$ we obtain

$$
p\left(x^{2}, \lambda, \tau\right) \int_{0}^{2 \pi} \omega\left(\frac{d x}{d s}, x\right) d s+q\left(x^{2}, \lambda, \tau\right) \int_{0}^{2 \pi} \omega\left(\frac{d x}{d s}, i x\right) d s=0
$$

We claim that the first integral is zero whereas the second integral is nonzero, which shows that $q$ vanishes identically. By the definitions of $c$ and $d$ in (9.1) we have $J d=c$, where $J$ is the matrix associated with the underlying symplectic form $\omega$. Therefore

$$
\begin{aligned}
& \int_{0}^{2 \pi} \omega\left(\operatorname{Re}\left(-i e^{-i s} v_{0}\right), \operatorname{Re}\left(e^{-i s} v_{0}^{*}\right)\right) d s=\pi \operatorname{Re}\left(\omega\left(-i v_{0}, \overline{v_{0}^{*}}\right)\right)=0, \quad \text { and } \\
& \int_{0}^{2 \pi} \omega\left(\operatorname{Re}\left(-i e^{-i s} v_{0}\right), \operatorname{Re}\left(i e^{-i s} v_{0}^{*}\right)\right) d s=\pi \operatorname{Re}\left(\omega\left(-v_{0}, \overline{v_{0}^{*}}\right)\right)=-\pi\left|v_{0}\right|^{2} \neq 0
\end{aligned}
$$


which proves the claim.

\section{Derivatives in the reduced system}

By the results of the previous section we know that the set of periodic solutions is locally given by solutions of the equation

$$
p\left(x^{2}, \lambda, \tau\right) x=0 .
$$

Theorem 7.1, which allows us to recognize the structure of the solution set of this equation in a neighborhood of $(0,0,0)$, follows directly from the next four lemmas.

Lemma $\mathbf{1 1 . 1}$

$$
p_{\tau}(0,0,0)=0
$$

Proof Observe that

$$
g_{x \tau}(0,0,0)=p_{\tau}(0,0,0)+i q_{\tau}(0,0,0) .
$$

Using the formulas in Golubitsky and Schaeffer [1985, p. 33], and the fact that $F_{\tau}=d F_{\tau}=\frac{d}{d s}$, we obtain at 0

$$
\begin{aligned}
g_{r \tau} & =\left\langle v_{0}^{*}, d F_{\tau} \cdot v_{0}-d^{2} F\left(v_{0}, L^{-1} P F_{\tau}\right)\right\rangle \\
& =\left\langle\operatorname{Re}\left(e^{-i s} v_{0}^{*}\right), \frac{d}{d s}\left(\operatorname{Re}\left(e^{-i s} v_{0}\right)\right)\right\rangle \\
& =0 \quad(\text { by }(9.2)) .
\end{aligned}
$$

Lemma 11.2

$$
p_{\lambda}(0,0,0)=-\frac{1}{2} \operatorname{Re}\left(v_{0}^{*} \cdot\left(d f_{\lambda}\right) v_{0}\right)
$$

Proof It follows from (8.3) that $F_{\lambda}(0,0)=0$. We compute

$$
\begin{aligned}
g_{x \lambda} & =\left\langle v_{0}^{*}, d F_{\lambda} \cdot v_{0}-d^{2} F\left(v_{0}, L^{-1} P F_{\lambda}\right)\right\rangle \\
& =-\left\langle v_{0}^{*}, d f_{\lambda} \cdot v_{0}\right\rangle \\
& =-\frac{1}{4}\left\langle v_{0}^{*}, d f_{\lambda} \cdot \overline{v_{0}}\right\rangle-\frac{1}{4}\left\langle\overline{v_{0}^{*}}, d f_{\lambda} \cdot v_{0}\right\rangle \\
& =-\frac{1}{2} \operatorname{Re}\left(v_{0}^{*} \cdot d f_{\lambda} \cdot v_{0}\right) .
\end{aligned}
$$

\section{Lemma 11.3}

$$
p_{\tau \tau}(0,0,0)=\overline{v_{0}^{*}} \cdot v_{0}^{*} .
$$

Proof This time we use formulas (3.37) and (3.38) in Golubitsky and Schaeffer [1985] and compute

$$
\begin{aligned}
g_{x \tau \tau} & =\left\langle v^{*}, d F_{\tau \tau} \cdot v+2 d F_{\tau} \cdot w\right\rangle \\
& =2\left\langle v^{*}, \frac{d}{d s} w\right\rangle \\
& =-2\left\langle\frac{d v^{*}}{d s}, w\right\rangle
\end{aligned}
$$

where $w$ is defined by

$$
w=-L^{-1} P d F_{\tau} \cdot v
$$


In particular, $w$ satisfies the ODE

$$
L w=-P \frac{d v}{d s}=-\frac{d v}{d s}
$$

The second equality holds since $\frac{d v}{d s} \in \mathcal{K}$ and since the kernel $\mathcal{K}$ is contained in the range $\mathcal{R}$ by the assumptions on $A_{0}$. We note that

$$
\frac{d v}{d s}=-\frac{i}{2}\left(e^{-i s} v_{0}-e^{i s} \bar{v}_{0}\right)
$$

and make the ansatz

$$
w(s)=\operatorname{Re}\left(e^{-i s} b_{1}\right) .
$$

Since $v=\operatorname{Re}\left(e^{-i s} v_{0}\right)$ it follows with (11.1) that $b_{1}$ has to sat isfy

$$
\left(A_{0}+i I\right) b_{1}=i v_{0}
$$

Hence, by the definition of $v_{0}$ and $v_{0}^{*}(9.1)$ and the action of $A_{0}$ on $v_{3}$ and $v_{4}(8.2)$, we conclude that $b_{1}=i v_{0}^{*}$ which yields

$$
\begin{aligned}
-2\left\langle\frac{d v^{*}}{d s}, w\right\rangle & =-\frac{1}{2}\left\langle-i v_{0}^{*}, i v_{0}^{*}\right\rangle-\frac{1}{2}\left\langle i \overline{v_{0}^{*}},-i \overline{v_{0}^{*}}\right\rangle \\
& =\frac{1}{2}\left(\left\langle i v_{0}^{*}, i v_{0}^{*}\right\rangle+\left\langle-i \overline{v_{0}^{*}},-i \overline{v_{0}^{*}}\right\rangle\right) \\
& =\operatorname{Re}\left(-i \overline{v_{0}^{*}} \cdot i v_{0}^{*}\right) \\
& =\overline{v_{0}^{*}} \cdot v_{0}^{*} .
\end{aligned}
$$

Therefore, $p_{r \tau}=\overline{v_{0}^{*}} \cdot v_{0}^{*}>0$, as desired.

\section{Lemma 11.4}

$$
p_{x^{2}}(0,0,0)=-\frac{3}{2} \operatorname{Re}\left[\overline{v_{0}^{*}} \cdot\left(\frac{1}{4} d^{3} f\left(v_{0}, v_{0}, \overline{v_{0}}\right)+d^{2} f\left(v_{0}, b_{0}\right)+d^{2} f\left(\overline{v_{0}}, b_{2}\right)\right)\right],
$$

where

$$
\begin{aligned}
A_{0} b_{0} & =\frac{1}{2} d^{2} f\left(v_{0}, \overline{v_{0}}\right) \\
\left(A_{0}-2 i I\right) b_{2} & =\frac{1}{4} d^{2} f\left(v_{0}, v_{0}\right) .
\end{aligned}
$$

Proof The proof of this lemma follows exactly the method of the derivation of the cubic term in the bifurcation equation at a Hopf bifurcation. We follow the proof of Proposition 3.3 in Golubitsky and Schaeffer [1985, pp. 352, 355-356].

Using Golulbitsky and Schaeffer [1985] (3.26), we have

$$
\frac{\partial p}{\partial\left(x^{2}\right)}=\left\langle v^{*} \cdot d^{3} F(v, v, v)+3 d^{2} F(v, w)\right\rangle
$$

where

$$
w=-L^{-1} P d^{2} F(v, v) .
$$

Observe that $d^{k} F=-d^{k} f$ when $k>1$. Recall that

$$
v^{\prime}=\frac{1}{2}\left(e^{-i s} v_{0}+e^{2 s} \overline{v_{0}}\right) \text { and } v^{*}=\frac{1}{2}\left(e^{-i s} v_{i 0}^{*}+e^{i s} \overline{v_{0}^{*}}\right) \text {. }
$$


Since $\left\langle e^{i m s}, e^{i n s}\right\rangle=0$ if $m \neq n$ it is easy to compute

$$
\left\langle v^{*},-d^{3} f(v, v, v)\right\rangle=-\frac{3}{8} \operatorname{Re}\left(\overline{v_{0}^{*}} \cdot d^{3} f\left(v_{0}, v_{0}, \overline{v_{0}}\right)\right)
$$

Similarly, one can verify that

$$
\left\langle v^{*}, d^{2} f(v, v)\right\rangle=0
$$

from which it follows that $d^{2} f(v, v) \in$ range $L$ and hence that $P d^{2} f(v, v)=d^{2} f(v, v)$. It also follows that we may assume

$$
w=b_{0}+e^{-2 i s} b_{2}+e^{2 i s} \overline{b_{2}},
$$

and we may use this form to solve

$$
L w=d^{2} f(v, v)
$$

for $b_{0} \in \mathbb{R}^{2 n}$ and $b_{2} \in \mathbb{C}^{2 n}$. Indeed,

$$
d^{2} f(v, v)=\frac{1}{4}\left\{e^{-2 i s} d^{2} f\left(v_{0}, v_{0}\right)+2 d^{2} f\left(v_{0}, \overline{v_{0}}\right)+e^{2 i s} d^{2} f\left(\overline{v_{0}}, \overline{v_{0}}\right)\right\}
$$

Combining the various terms, we have that

$$
\left\langle v^{*}, d^{2} F(v, w)\right\rangle=-\frac{1}{2} \operatorname{Re}\left[\overline{v_{0}^{*}} \cdot\left(d^{2} f\left(v_{0}, b_{0}\right)+d^{2} f\left(\overline{v_{0}}, b_{2}\right)\right)\right],
$$

where

$$
A_{0} b_{0}=\frac{1}{2} d^{2} f\left(v_{0}, \overline{v_{0}}\right)
$$

and

$$
\left(A_{0}-2 i I\right) b_{2}=\frac{1}{4} d^{2} f\left(v_{0}, v_{0}\right)
$$

\section{Singularity theory}

Having proved Theorem 7.1 we follow van der Meer [1985] in viewing the bifurcation equation (7.1) as a bifurcation problem in the perturbed period parameter $\tau$ with imperfection parameter $\lambda$. We follow Bridges [1990] in solving $p=0$ by using $\mathbb{Z}_{2}$-equivariant singularity theory. Setting $\lambda=0$ in (7.1) and using the facts

$$
p(0)=0, \quad p_{\tau}(0)=0, \quad p_{\tau \tau}(0)>0 \quad \text { and } \quad p_{x^{2}}(0) \neq 0
$$

proved in Theorem 7.1 , we can apply the determinacy results from $\mathbb{Z}_{2}$-equivariant singularity theory with a distinguished parameter (Proposition 3.4(b) Golubitsky and Schaeffer [1985], p. 259) to show that (7.1) with $\lambda=0$ is $\mathbb{Z}_{2}$-equivalent to

$$
\left( \pm x^{2}+\tau^{2}\right) x=0 \text {. }
$$

Next we use the $\mathbb{Z}_{2}$ universal unfolding theorem (Theorem 3.3 of Golubitsky and Schaeffer [1985], p. 259) and the fact that $p_{\lambda}(0) \neq 0$ to prove that the reduced bifurcation equation (7.1) is $\mathbb{Z}_{2}$-equivalent to the normal form (7.2).

\section{Part III Periodic Orbits near Resonances}

In this part, we study two questions: how to find periodic solutions in reversible systems near equilibria in $k: \ell$ resonance and how to find periodic solutions in Hamiltonian systems near equilibria with $k: \ell$ resonances. The answer to the first 
question relies on using symmetry - both the $\mathbf{S}^{\mathbf{1}}$-symmetry of phase shift and the reversible symmetry - and (a slightly nonstandard) singularity theory to solve the reduced equations obtained through Liapunov-Schmidt reduction. We use implicit constraints and symmetry to reduce the answer for the second question to the answer for the first question.

These subjects have been well studied and we now describe the relation of the present approach to $k: \ell$ resonances in reversible and Hamiltonian systems with that of other authors. The survey in Arnol'd [1988] applies directly only to systems that are in Birkhoff normal form. Although, as is remarked there, a great deal can subsequently be deduced for systems that are subject to higher order perturbations, these deductions are not made explicit. Duistermaat [1983], whose work is largely based on Schmidt [1974], obtains similar results to ours but the singularity-theoretic calculations are more delicate because he works in a different context with a different equivalence relation. Consequently, his normal forms have one extra modal parameter for $k: 1$ resonances with $k \geq 3$. In compensation, he obtains more delicate information, such as stabilities. A reference for the reversible case is Sevryuk [1988], but again the singularity theory is treated differently.

There are many earlier papers, including Palmore [1969], Meyer and Palmore [1970], Meyer and Schmidt [1971], Roels [1971a, b], Henrard [1973], Schmidt and Sweet [1973], Sweet [1973] and Schmidt [1973, 1978]. They differ from the present work in method - most of them use perturbation expansions and prove convergence, although Schmidt and Sweet [1973] and Sweet [1973] use the 'alternative method' - which is essentially the same as Liapunov-Schmidt reduction. Moreover, the results of Sweet [1973] apply to any system with a first integral, not just a Hamiltonian system. A good general source for the related method of averaging and further references, is Sanders and Verhulst [1985].

The most important difference with other works and the present one, is that in this paper we obtain normal forms that include the effect of a 'detuning parameter'. Of the references cited, only Schmidt [1973], Duistermaat [1983], and Arnol'd [1988] do this: all others consider only the dynamics at resonance. Moreover, we find all $k, \ell$, and 1-families of periodic solutions near equilibrium, and our results apply to reversible systems as well as to Hamiltonian ones. Our singularity-theoretic methods differ considerably from those of all authors except Duistermaat [1983] and Arnol'd [1988]. The latter gives no details about this method, but it is clear that singularity theory is involved.

Another more technical difference is that most of the above authors assume that the Hamiltonian is real analytic - a property that is essential for the series methods they employ. The exceptions are Arnol'd [1988], Duistermaat [1983] and Roels [1971a]; the latter assumes that the Hamiltonian is kinetic plus potential where the potential is $C^{1}$ and has locally Lipschitz gradient.

Most authors identify which coefficients in the Hamiltonian are relevant and which nondegeneracy conditions must hold. Some obtain stability information, which we do not attempt here, though the methods of Montaldi, Roberts, and Stewart [1990b] could in principle be used and the calculations would be very similar to those of Duistermaat [1983].

For examples of reversible systems in mechanics that may or may not be Hamiltonian, and their stability and bifurcation properties, we refer to O'Reilly, Malhotra and Namachchivaya [1993] and references therein. 
Another useful tool in the study of resonances is that of pinched spheres and invariants. When a resonant system is put into normal form, the Hamiltonian acquires an $\mathbf{S}^{\mathbf{l}}$ symmetry, which can be used to perform reduction, leading to an analysis of the normal form. The reduced spaces are spheres for the 1:1 resonance and are 'pinched spheres' for higher order resonances. These spheres carry an interesting Lie-Poisson type of Poisson structure. The 'pinches' come about because of the isotropy of special points that occurs for higher order resonances. The systems on these spheres can often be analyzed rather effectively. References for this approach are Kummer [1979], Cushman and Rod [1982], Iwai [1985], Kummer [1990], David, Holm, and Tratnick [1990] and references therein. Knobloch, Mahalov, and Marsden [1993] contains an exposition of the Poisson structure of this problem.

Of course, the methods above often can be extended to multiple resonances. We do not discuss this aspect here, except for the obvious remark that our methods are not restricted to simple resonances. Some of the relevant literature is Duistermaat [1984], Sanders and Verhulst [1985] (and references therein), Hoveijn and Verhulst [1990], Haller and Wiggins [1992], and Haller [1993] (and references therein).

\section{Preliminaries}

We assume that the state space is $\mathbb{R}^{2 n}$. Many different 'time-reversal' symmetries are possible in a general system of ODEs; here we consider only the timereversal symmetry that takes the form

$$
R(x, y)=(x,-y)
$$

where $x, y \in \mathbb{R}^{n}$. Abstractly, we write the system of ODEs as

$$
\frac{d X}{d s}=f(X, \lambda)
$$

where $X=(x, y) \in \mathbb{R}^{2 n}$ and $\lambda \in \mathbb{R}$. We assume that (13.2) has an equilibrium at $\lambda=0$ which is $R$-invariant in the time-reversible case, and which without loss of generality we take to be the origin; that is,

$$
f(0,0)=0 .
$$

Let $A_{0}=d f_{0.0}$ be the Jacobian matrix of this equilibrium and let $0<k<\ell$ be coprime integers. Assume

(R1) $\pm k i$ and $\pm \ell i$ are simple eigenvalues of $A_{0}$ and $\pm m i$ is not an eigenvalue of $A_{0}$ where $m \neq k, \ell$ is any nonnegative integer.

Hypothesis (R1) implies that $A_{0}$ is invertible, so by the implicit function theorem there is a unique branch of equilibria $X(\lambda)$ with $X(0)=0$. Therefore

$$
f(X(\lambda), \lambda) \equiv 0 \text {. }
$$

For Hamiltonian systems we can change coordinates directly in $X$ to assume $X(\lambda) \equiv$ 0 and

$$
f(0, \lambda) \equiv 0 .
$$


Slightly more care is needed to arrive at the same result in the time-reversible case. Recall that time-reversibility implies

$$
\begin{aligned}
f(R X, \lambda) & =-R f(X, \lambda) \\
A_{0} R & =-R A_{0} .
\end{aligned}
$$

Hence

$$
f(R X(\lambda), \lambda) \equiv 0,
$$

and uniqueness of solutions obtained by the implicit function theorem implies that

$$
R X(\lambda)=X(\lambda)
$$

Now a change of coordinates that preserves time-reversibility leads to (13.3).

At resonance we may always factor out common denominators and rescale time so that the resonant eigenvalues are integer multiples of $i$, and we assurne from now on that this has been done. In Hamiltonian systems it is well known that generically purely imaginary eigenvalues can pass through resonances as a parameter is varied. To verify the corresponding statement for time-reversible systems, we must show that eigenvalues in these systems are also locked onto the imaginary axis. This is also well known - but less well known - so we now give details.

In so doing, we discuss the reasonableness of the assumption (13.1) as well as its consequences. Let $V^{+}=\{(x, 0)\}$ be the +1 eigenspace of $R$ and let $V^{-}=\{(0, y)\}$ be the -1 eigenspace of $R$. The identity (13.5) implies that

$$
A_{0}: V^{+} \rightarrow V^{-} \text {and } A_{0}: V^{-} \rightarrow V^{+} .
$$

Observe that $V^{+}$and $V^{-}$are invariant subspaces under $A_{0}^{2}$. Hypothesis (R1) implies that $A_{0}$ is invertible, so that $A_{0}^{2} \mid V^{+}$and $A_{0}^{2} \mid V_{-}$are similar matrices, that is,

$$
A_{0}^{2} \mid V^{+}=\left(A_{0} \mid V^{+}\right)^{-1}\left(A_{0}^{2} \mid V^{-}\right)\left(A_{0} \mid V^{+}\right) \text {. }
$$

Hence these restrictions have the same eigenvalues. It follows that the eigenvalues of $A_{0}$ are precisely the square roots of the eigenvalues of $A_{0}^{2} \mid V^{+}$, and the eigenvalues of $A_{0}$ fall into three classes:

(i) $\pm \sigma ; \quad \sigma>0$,

(ii) $\pm \mu i ; \quad \mu>0$,

(iii) $\pm \sigma \pm \mu i ; \quad \sigma, \mu>0$,

corresponding to whether the eigenvalues of $A_{0}^{2} \mid V^{+}$are positive, negative or complex. It follows that the existence of purely imaginary eigenvalues of $A_{0}$ is generic.

We now show that the resonance hypothesis (R1) is a codimension one assumption in time-reversible systems. Let

$$
A_{\lambda}=d f_{0, \lambda}
$$

It follows that as $\lambda$ is varied the purely imaginary eigenvalues can (generically be expected to) pass through $k: \ell$ resonances with eigenvalues $\pm k i, \pm \ell i$ when $\lambda=0$. Generically these eigenvalues are simple. By rescaling time we can assume that the appropriate eigenvalues of $A_{\lambda}$, for all $\lambda$ near zero, are $\pm k i$ and $\pm \omega(\lambda) i$ where $\omega$ is smooth in $\lambda$ and $\omega(0)=\ell$. We assume that $A_{0}$ has no other resonant eigenvalues $\pm m i$, where $m \neq k, \ell$ is a non-negative integer; and that the critical eigenvalues pass through resonance with nonzero speed, that is, 
(R2) $\omega^{\prime}(0) \neq 0$.

It is well known that $\mathbf{S}^{1}$ has two families of nontrivial symplectic representations, in which

$$
\theta \cdot z=e^{i i \theta} z
$$

or

$$
\theta \cdot z=e^{-\ell i 0} z
$$

where $\ell \geq 1, z \in \mathbb{C}$, and $\mathbb{C}$ carries its standard symplectic structure. Call these representations $\rho_{\ell}$ and $\rho_{-\ell}$. The map $z \mapsto \bar{z}$ shows that $\rho_{\ell}$ and $\rho_{-\ell}$ are isomorphic as abstract representations. However, this isomorphism does not preserve the symplectic structure - see Montaldi, Roberts and Stewart [1988] and Dellnitz and Melbourne [1993]. It is usual to distinguish between these two representations by referring to a $k: \ell$ resonance or a $k:-\ell$ resonance. More precisely, let $\left(z_{1}, z_{2}\right)$ be complex coordinates on $\mathbb{C}^{2}$. Without loss of generality the $S^{1}$-action on $z_{1}$ is by $\rho_{k}$ where $k \geq 1$. Then the $S^{1}$-action on $z_{2}$ is either $\rho_{\ell}$ or $\rho_{-\ell}$ where $\ell \geq 1$. The first case is a $k: \ell$ resonance and the second a $k:-\ell$ resonance.

\section{Normal forms with $S^{1}$-Symmetry}

We now find periodic solutions to (13.2) with period approximately $2 \pi$ using a Liapunov-Schmidt reduction. Let

$$
F: C_{2 \pi}^{1} \times \mathbb{R} \times \mathbb{R} \rightarrow C_{2 \pi}
$$

be defined by

$$
F(u(s), \lambda, \tau)=(1+\tau) \frac{d u}{d s}-f(u(s), \lambda),
$$

and solve $F=0$ by Liapunov-Schmidt reduction in the standard way. The resonance hypothesis $(R 1)$ implies that the kernel of $d F_{0,0,0}$ is four-dimensional. Thus we can write the reduced equation as

$$
g: \mathbb{C}^{2} \times \mathbb{R} \times \mathbb{R} \rightarrow \mathbb{C}^{2}
$$

and solving $g(z, \lambda, \tau)=0$ yields all small-amplitude periodic solutions of (13.2) with period near $2 \pi$.

The $\mathbf{S}^{1}$ phase shift symmetry that acts on periodic functions in $\mathcal{C}_{2 \pi}$ restricts the form of $g$ considerably. Our eigenvalue assumptions imply that on the above kernel, $\theta \in \mathbf{S}^{1}$ acts by

$$
\theta\left(z_{1}, z_{2}\right)=\left(e^{k i \theta} z_{1}: e^{\ell i \theta} z_{2}\right)
$$

where $k \geq 1$ and $\ell$ (which may be positive or negative) are coprime and $g$ commutes with this action. A calculation from invariant theory determines the general form of $g$. We state the result for the $k: \ell$ resonance in the following lemma, and discuss the $k:-\ell$ resonance afterwards.

Lemma 14.1 Assume that $g: \mathbb{C}^{2} \rightarrow \mathbb{C}^{2}$ is equivariant with respect to the $k: \ell$ action of $\mathbf{S}^{1}$. Then

$$
g\left(z_{1}, z_{2}\right)=\left(P_{1} z_{1}+Q_{1}{\overline{z_{1}}}^{\ell-1} z_{2}^{k}, P_{2} z_{2}+Q_{2} z_{1}^{\ell}{\overline{z_{2}}}^{k-1}\right)
$$

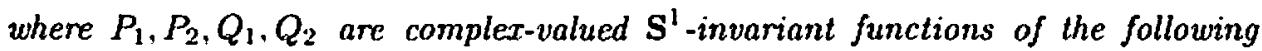
three of the four $\mathbf{S}^{1}$-invariants

$$
\left|z_{1}\right|^{2},\left|z_{2}\right|^{2}, \operatorname{Re}\left(z_{1}^{\ell}{\overline{z_{2}}}^{k}\right)
$$


Moreover, $P_{1}, P_{2}, Q_{1}, Q_{2}$ are uniquely determined by $g$.

Proof Write $g=\left(g_{1}, g_{2}\right)$ in coordinates. We derive the form of $g_{1}$; the derivation for $g_{2}$ is similar. Equivariance implies that

$$
g_{1}\left(z_{1}, z_{2}\right)=e^{-k i \theta} g\left(e^{k i \theta} z_{1}, e^{i i \theta} z_{2}\right)
$$

Write the Taylor series for $g_{1}$ as

$$
g_{1}\left(z_{1}, z_{2}\right)=\sum A_{a b c d} z_{1}^{a}{\overline{z_{1}}}^{b} z_{2}^{c}{\overline{z_{2}}}^{d}
$$

and use the invariance condition to show that the only terms that survive are the ones for which

$$
k(a-b-1)+\ell(c-d)=0 .
$$

Collect terms in the Taylor expansion of $g_{1}$ that have a common factor of $z_{1} \overline{z_{1}}$ or $z_{2} \overline{z_{2}}$ to write $g_{1}$ in the form

$$
g_{1}\left(z_{1}, z_{2}\right)=\sum B_{a b c d}\left(\left|z_{1}\right|^{2},\left|z_{2}\right|^{2}\right) z_{1}^{a} \bar{z}_{1}^{b} z_{2}^{c}{\overline{z_{2}}}^{d},
$$

where two of the indices $a, b, c, d$ are zero. Since $k$ and $\ell$ are coprime and nonnegative we conclude that

$$
g_{1}\left(z_{1}, z_{2}\right)=A\left(\left|z_{1}\right|^{2},\left|z_{2}\right|^{2}, z_{1}^{k}{\overline{z_{2}}}^{k}\right) z_{1}+B\left(\left|z_{1}\right|^{2},\left|z_{2}\right|^{2},{\overline{z_{1}}}^{k} z_{2}^{k}\right){\overline{z_{1}}}^{\ell-1} z_{2}^{k} .
$$

We now assert that $g_{1}$ can be put into the form

$$
g_{1}\left(z_{1}, z_{2}\right)=C\left(\left|z_{1}\right|^{2},\left|z_{2}\right|^{2}, \operatorname{Re}\left(z_{1}^{\ell}{\overline{z_{2}}}^{k}\right)\right) z_{1}+D\left(\left|z_{1}\right|^{2},\left|z_{2}\right|^{2}, \operatorname{Re}\left(z_{1}^{\ell}{\overline{z_{2}}}^{k}\right)\right) \overline{z_{1}}{ }^{\ell-1} z_{2}^{k} \text {. }
$$

To verify this assertion observe that

$$
\begin{aligned}
\left(z_{1}^{\ell}{\overline{z_{2}}}^{k}\right)^{m} z_{1} & =\left(\left(z_{1}^{\ell}{\overline{z_{2}}}^{k}+{\overline{z_{1}}}^{\ell} z_{2}^{k}\right)-{\overline{z_{1}}}^{\ell} z_{2}^{k}\right)^{m} z_{1} \\
& =\sum_{i+j=m} k_{i j}\left[\operatorname{Re}\left(z_{1}^{\ell}{\overline{z_{2}}}^{k}\right)\right]^{i}\left({\overline{z_{1}}}^{\ell} z_{2}^{k}\right)^{j} z_{1} .
\end{aligned}
$$

Then note that when $j>0$

$$
\left(\bar{z}_{1}^{k} z_{2}^{k}\right)^{j} z_{1}=\left(\bar{z}_{1}^{k} z_{2}^{k}\right)^{j-1}\left|z_{1}\right|^{2} \bar{z}_{1}^{\ell-1} z_{2}^{k}
$$

Similarly,

$$
\begin{aligned}
& \left({\overline{z_{1}}}^{\dagger} z_{2}^{k}\right)^{m}{\overline{z_{1}}}^{(-1} z_{2}^{k}=\left(\left({\overline{z_{1}}}^{\ell} z_{2}^{k}+z_{1}^{\ell}{\overline{z_{2}}}^{k}\right)-z_{1}^{\ell}{\overline{z_{2}}}^{k}\right)^{m}{\overline{z_{1}}}^{l-1} z_{2}^{k} \\
& =\sum_{i+j=m} k_{i j}\left[\operatorname{Re}\left(z_{1}^{\ell}{\overline{z_{2}}}^{k}\right)\right]^{i}\left(z_{1}^{\ell}{\overline{z_{2}}}^{k}\right)^{j}{\overline{z_{1}}}^{(-1} z_{2}^{k} \text {. }
\end{aligned}
$$

Then note that when $j>0$,

$$
\left(z_{1}^{\ell}{\overline{z_{2}}}^{k}\right)^{j}{\overline{z_{1}}}^{\ell-1} z_{2}^{k}=\left(z_{1}^{\ell} \bar{z}_{2}^{k}\right)^{j-1}\left|z_{1}\right|^{2 \ell-2}\left|z_{2}\right|^{2 k} z_{1} .
$$

A double induction completes the proof of the assertion.

To complete the proof of the lemma we verify that the invariant functions $P_{1}$, $P_{2}, Q_{1}, Q_{2}$ are unique. To see this assume that

$$
P_{1} z_{1}+Q_{1}{\overline{z_{1}}}^{\ell-1} z_{2}^{k} \equiv 0 \text {. }
$$

Using $\mathbf{S}^{\prime}$ symmetry we can assume that $z_{1}=x_{1}$ is real and obtain

$$
P_{\mathrm{i}} x_{1}+Q_{1} x_{1}^{\ell-1} z_{2}^{k} \equiv 0 .
$$


Since $P_{1}$ and $Q_{1}$ are clearly invariant under the action $z_{2} \mapsto \overline{z_{2}}$ it follows that

$$
P_{1} x_{1}+Q_{1} x_{1}^{\ell-1}{\overline{z_{2}}}^{k} \equiv 0
$$

Subtracting (14.3) from (14.2) yields

$$
Q_{1} x_{1}^{\ell-1} \operatorname{Im}\left(z_{2}^{k}\right) \equiv 0
$$

so that $Q_{1} \equiv 0$, and hence $P_{1} \equiv 0$ as desired.

For the $k:-\ell$ resonance the results are similar, but $z_{2}$ must be replaced by $\overline{z_{2}}$ and conversely. Clearly zeros of (14.1) are in one-to-one correspondence with zeros of the corresponding mapping with $z_{2}$ and $\overline{z_{2}}$ interchanged, so the zeros for the $k:-\ell$ resonance can be read off from those for the corresponding $k: \ell$ resonance. When seeking periodic orbits of the original Hamiltonian system, determined by these zeros, we may therefore confine attention to the $k: \ell$ resonance. We do so from now on.

\section{Reduced equations in the time-reversible case}

The form of $g$ is further restricted by time-reversibility. It can be checked that the time-reversal symmetry $R$ preserves the kernel and cokernel of $d F$ and hence anticommutes with $g$, that is,

$$
g\left(\overline{z_{1}}, \overline{z_{2}}\right)=-\overline{g\left(z_{1}, z_{2}\right)} .
$$

This anticommutativity allows us prove a stronger version of Lemma 14.1.

Lemma 15.1 Time-reversal symmetry implies that the invariant functions $P_{1}$, $P_{2}, Q_{1}, Q_{2}$ are purely imaginary.

Set $P_{j}=i P_{j}^{i}$ and $Q_{j}=i Q_{j}^{i}$. After dividing by $i$ we see that finding periodic solutions to the differential equation (13.2) is equivalent to finding solutions to the following system of two complex equations:

$$
\begin{aligned}
& P_{1}^{i} z_{1}+Q_{1}^{i} \bar{z}_{1}^{\ell-1} z_{2}^{k}=0 \\
& P_{2}^{i} z_{2}+Q_{2}^{i} z_{1}^{i} \bar{z}_{2}^{k-1}=0
\end{aligned}
$$

where $P_{1}^{i}, P_{2}^{i}, Q_{1}^{i}, Q_{2}^{i}$ are real-valued functions of $\left|z_{1}\right|^{2},\left|z_{2}\right|^{2}, \operatorname{Re}\left(z_{1}^{i} \bar{z}_{2}^{k}\right), \lambda$ and $\tau$.

We begin by finding the one-parameter family of periodic solutions that is always present by virtue of the Liapunov center theorem (for time-reversible systems). Suppose that $z_{1}=0$. Then (15.1) is satisfied and (15.2) reduces to $P_{2}^{i} z_{2}=0$. Thus either $z_{2}=0$ (the trivial equilibrium) or $P_{2}^{i}=0$. Since $P_{2.5}^{i}(0) \neq 0$ we can use the implicit function theorem to solve $P_{2}^{i}=0$ for $\tau$ and obtain a family of periodic solutions with period near $2 \pi / \ell$. We refer to this family as the $\ell$-family of solutions. Since $\ell>k$ the eigenvalues $\pm \ell i$ are nonresonant.

We may now assume that $z_{1} \neq 0$. Applying the $\mathbf{S}^{1}$ action we further assume that $z_{1}=x_{1}>0$ where $x_{1} \in \mathbb{R}$. Dividing by $x_{1}$ in (15.1), shows that the remaining periodic solutions may be found by solving

$$
\begin{aligned}
P_{1}^{i}+Q_{1}^{i} x_{1}^{k-2} z_{2}^{k} & =0 \\
P_{2}^{i} z_{2}+Q_{2}^{i} x_{1}^{i} z_{2}^{k-1} & =0 .
\end{aligned}
$$


Solving the imaginary part of (15.3) leads to the equation $Q_{1}^{i} \operatorname{Im}\left(z_{2}^{k}\right)=0$. Since $Q_{\mathrm{I}}^{i}$ is the coefficient of a higher order term in the reduced equation (a term of order at least two) we can make the genericity hypothesis:

(R3) $Q_{1}^{i}(0) \neq 0$.

It follows from (R3) that

$$
\operatorname{Im}\left(z_{2}^{k}\right)=0
$$

at solutions.

Next, since $P_{1 . \tau}^{i}(0) \neq 0$, we can use the implicit function theorem to solve the real part of (15.3) for $\tau$ and then substitute this solution for $\tau$ in (15.4). Thus finding the desired families of periodic solutions reduces to solving (15.4).

Equation (15.5) implies that $z_{2}^{k} \in \mathbb{R}$, that is,

$$
z_{2}=x_{2} e^{m i \pi / k}
$$

where $m$ is an integer, $x_{2} \in \mathbb{R}$ and $x_{2} \geq 0$. Now the group generated by $e^{2 \pi i / k} \in \mathbf{S}^{1}$ fixes $x_{1}$, so up to symmetry (15.6) reduces to two cases

$$
\begin{aligned}
& z_{2}=x_{2} \\
& z_{2}=x_{2} \mathrm{e}^{i \pi / k} .
\end{aligned}
$$

It follows that $\left|z_{2}\right|^{2}=x_{2}^{2}$ and $z_{2}^{k}=\epsilon x_{2}^{k}$ where $\epsilon= \pm 1$.

We divide our analysis into two cases: $k>1$ and $k=1$. However, note that when $k$ is odd, we could have used the discrete symmetries to conjugate $z_{2}$ to $x_{2} \in \mathbb{R}$; that is, we can permit $x_{2}$ to be negative as well as positive. This has the advantage of eliminating the parameter $\epsilon= \pm 1$ from the problem. We will use this observation when considering the case $k=1$.

The case $k>1$ When $k>1$ we can set $z_{2}=0$ to obtain a family of solutions directly, because (15.4) is automatically satisfied. Equation (15.3) (which we solved previously for $\tau$ ) now reduces to $P_{1}^{i}=0$. We call this the $k$-family of solutions. It consists of solutions with period near $2 \pi / k$ and is given by the nonresonant eigenvalues $\pm k i$ in the Liapunov center theorem.

Next we assume that $z_{2} \neq 0$ and multiply (15.4) by $\overline{z_{2}}$ to get

$$
P_{2}^{i}\left|z_{2}\right|^{2}+Q_{2}^{i} x_{1}^{\ell} \bar{z}_{2}^{k}=0
$$

The imaginary part of (15.7) is just

$$
Q_{2}^{i} \operatorname{Im}\left(z_{2}^{k}\right)=0
$$

which holds by (15.5). Indeed, (15.7) may be rewritten as

$$
P_{2}^{i} x_{2}^{2}+\epsilon Q_{2}^{i} x_{1}^{\ell} x_{2}^{k}=0 .
$$

Since $x_{2} \neq 0$ and $k \geq 2$ we may divide by $x_{2}^{2}$ to obtain

$$
P_{2}^{i}+\epsilon Q_{2}^{i} x_{1}^{\ell} x_{2}^{k-2}=0,
$$

where $\ell \geq 3$ 
Since $P_{2}^{i}$ and $Q_{2}^{i}$ are functions of $x_{1}^{2}, x_{2}^{2}, \epsilon x_{1}^{\varepsilon} x_{2}^{k}, \lambda, \tau$ and $\tau=\tau\left(x_{1}^{2}, x_{2}^{2}, \epsilon x_{1}^{\ell-2} x_{2}^{k}, \lambda\right)$, it follows that (15.8) has the form

$$
p^{c}\left(x_{1}, x_{2}, \lambda\right)=r^{\epsilon}\left(x_{1}^{2}, x_{2}^{2}, \lambda\right)+s^{\epsilon}\left(x_{1}^{2}, x_{2}^{2}, \lambda\right) x_{1}^{\ell-2} x_{2}^{\lambda-2}
$$

where $s^{\epsilon}(0,0, \lambda)=0$ and $\epsilon= \pm 1$ yields two distinct functions $p^{\ell}$. It may be verified that the lowest order terms in $r^{c}$ do not depend on $\epsilon$, that is,

$$
r^{c}\left(x_{1}^{2}, x_{2}^{2}, \lambda\right)=a x_{1}^{2}+b x_{2}^{2}+c \lambda+\ldots
$$

where $a, b, c$ do not depend on $\epsilon$. (The lowest order terms in $s^{t}$ do depend on $\epsilon$.) solve

The case $k=1$ When $k=1$ the previous discussion implies that we must

$$
P_{2}^{i} x_{2}+Q_{2}^{i} x_{1}^{\ell}=0
$$

where $P_{2}^{i}, Q_{2}^{i}$ are functions of $x_{1}^{2}, x_{2}^{2}, x_{1}^{\ell} x_{2}, \lambda, \tau$, and $\tau=\tau\left(x_{1}^{2}, x_{2}^{2}, x_{1}^{\ell-2} x_{2}, \lambda\right)$. Since $k=1$ is odd, we may assume $x_{2} \in \mathbb{R}, x_{2} \neq 0$ rather than considering the two cases $\epsilon= \pm 1$. Also, note that generically (when $Q_{2}(0) \neq 0$ ) there is no 1-family when $x_{2}=0$ (since $x_{2}=0$ then implies that $x_{1}=0$ ). However, we will find 1-families of solutions when $x_{2} \neq 0$.

As in the case $k>1$ we can rewrite (15.10) uniquely as

$$
p\left(x_{1}, x_{2}, \lambda\right)=r\left(x_{1}^{2}, x_{2}^{2}, \lambda\right) x_{2}+s\left(x_{1}^{2}, x_{2}^{2}, \lambda\right) x_{1}^{\ell-2}=0
$$

where $s(0,0, \lambda)=0$.

\section{Reduced equations in the Hamiltonian case}

In this section we show that at a point of $k: \ell$ resonance in a Hamiltonian system we can also find time-periodic solutions by solving a system analogous to the one used to find time-periodic solutions in the reversible case. To establish this, we control the Liapunov-Schmidt reduced equations by using the extra structure imposed by the Hamiltonian character instead of using the time-reversal symmetry, as in Lemma 15.1.

We consider the equation $\dot{u}=f(u)=X_{H}(u)$ on a symplectic vector space isomorphic to $\mathbb{R}^{2 n}=\mathbb{C}^{n}$, as before (later we will add parameters) and make assumptions similar to the reversible case:

(RH1) $x_{0}$ is a fixed point: $f\left(x_{0}\right)=0$. Let $A_{0}=d f\left(x_{0}\right)$ be the linearization at $x_{0}$ and let $k$ and $\ell$ be coprime positive integers.

(RH2) $\pm k \omega_{0} i$ and $\pm \ell \omega_{0} i$ are simple eigenvalues of $A_{0}$ and $p \omega_{0} i$ is not an eigenvalue for $p$ a nonnegative integer other than $k$ or $\ell$. Let $v_{k}$ and $v_{k}$ be the eigenvectors of $A_{0}$ for the eigenvalues $k \omega_{0} i$ and $\ell \omega_{0} i$ respectively.

By (RH1) the function $H$ has a critical point at the point $x_{0}$. By (RH2) the second derivative is nondegenerate on the four-dimensional eigenspace.

We can assume that $0<k<\ell$, as in the reversible case, and that $\omega_{0}=1$ by rescaling time. Now apply the Liapunov-Schmidt procedure to the map $F$. Let. $\mathcal{X}=\mathcal{C}_{2 \pi}^{1} \times \mathbb{R}$ and $\mathcal{Y}=\mathcal{C}_{2 \pi}^{0}$ (together with bifurcation parameters to be included later). Let. $\mathrm{S}^{1}$ act on the first two components of $\mathcal{X}$ by $(\theta \cdot u)(s)=u(s+\theta)$ and similarly on $\mathcal{Y}$. Note that $F$ is $\mathbf{S}^{1}$-equivariant. 
The derivative of $F$ at $(u, \tau)=\left(x_{0}, 0\right)$ is $L$, where

$$
L v \equiv d F\left(x_{0}, 0\right) \cdot v=\frac{d v}{d s}-A_{0} v .
$$

The kernel of $d F\left(x_{0}, 0\right)$ coincides in this case (unlike Hamiltonian Hopf) with the eigenspace of the four eigenvalues and therefore is symplectic and is even the symplectic sum of two two-dimensional symplectic subspaces. Thus, we will proceed by using the condition (S). By (RH2), $\operatorname{ker} d F\left(x_{0}, 0\right)$ is spanned by the four periodic functions

$$
\operatorname{Re}\left(e^{i k s} v_{k}\right), \operatorname{Im}\left(e^{i k s} v_{k}\right), \operatorname{Re}\left(e^{i \ell s} v_{\ell}\right), \operatorname{Im}\left(e^{i k s} v_{\ell}\right)
$$

Exactly as in our treatment of the Liapunov center theorem, we can use the complex structure already on phase space to rephrase this as the space spanned by the loops $e^{i k s} z v_{k}$ and $e^{i t s} z v_{i}$. Thus we can identify the kernel of $d F$ with $\mathbb{C}^{2}$; explicitly,

$$
\left(z_{1}, z_{2}\right) \mapsto\left(z_{1} e^{i k s} v_{k}, z_{2} e^{i \ell s} v_{\ell}\right)
$$

The same calculation as in the case of the Liapunov center theorem shows that the symplectic structure on the kernel coincides with the standard symplectic structure on $\mathbb{C}^{2}$. We may choose coordinates on $\mathbb{C}^{2}$ so that the matrix $A_{0}$ has the block form

$$
\left(\begin{array}{cccc}
0 & k & 0 & 0 \\
-k & 0 & 0 & 0 \\
0 & 0 & 0 & \ell \\
0 & 0 & -\ell & 0
\end{array}\right)
$$

and the second derivative $d^{2} H(0)$ is

$$
\left(\begin{array}{llll}
\alpha & 0 & 0 & 0 \\
0 & \alpha & 0 & 0 \\
0 & 0 & \beta & 0 \\
0 & 0 & 0 & \beta
\end{array}\right)
$$

where $\alpha$ and $\beta$ are nonzero constants. In these coordinates, the components of $v_{k}$ are $(1, i, 0,0,0, \ldots, 0)$ and those of $v_{\ell}$ are $(0,0,1, i, 0, \ldots, 0)$.

Because condition (S) holds, the kernel of $L^{*}$ equals the kernel of $L$ and is isomorphic to $\mathbb{C}^{2}$ by means of

$$
\left(z_{1}, z_{2}\right) \mapsto\left(z_{1} e^{i k s} v_{k}^{*}, z_{2} e^{i t s} v_{f}^{*}\right)
$$

where $v_{k}^{*}$ is the eigenvector for $A_{0}^{*}$ given by $A_{0}^{*} v_{k}^{*}=-i k^{*}$ and similarly for $v_{\hat{\varepsilon}}^{*}$. The first four components of $v_{k}^{*}$ and $v_{k}$ are the same, as are those of $v_{i}^{*}$ and $v_{i}$.

The Liapunov-Schmidt procedure in the context of assumption (S), may now be applied. Recall that we use the standard inner product on $\mathbb{C}^{n}$, and that $L^{*} v=-v^{\prime}-A_{0}^{*} v, \mathcal{M}=\operatorname{range} L^{*}, \mathcal{K}=\operatorname{ker} L$, and $\mathcal{N}=\operatorname{ker} L^{*}$. The bifurcation equation for $F$ has the form

$$
g: \mathbb{C}^{2} \times \mathbb{R} \times \mathbb{R} \rightarrow \mathbb{C}^{2}
$$

and, being $S^{1}$-equivariant, $g$ has the form

$$
\begin{aligned}
& g_{1}=P_{1} z_{1}+Q_{1}{\overline{z_{1}}}^{k-1} z_{2}^{k}, \\
& g_{2}=P_{2} z_{2}+Q_{2} z_{1}^{\ell}{\overline{z_{2}}}^{k-1} .
\end{aligned}
$$


Of course, the bifurcation equations will be $g=0$. By Theorem 6.2, the mapping $g$ is a Hamiltonian vector field with an $\mathbf{S}^{1}$-invariant Hamiltonian $H$. Being invariant, the function $H$ can be written as a function of the invariants which we shall denote $a=z_{1} \overline{z_{1}}, b=z_{2} \overline{z_{2}}, c=\frac{1}{2}\left(z_{1}^{\ell}{\overline{z_{2}}}^{k}+{\overline{z_{1}}}^{\ell} z_{2}^{k}\right), d=-\frac{i}{2}\left(z_{1}^{\ell} \bar{z}^{k}-{\overline{z_{1}}}^{\ell} z_{2}^{k}\right)$, and the parameters $\lambda$ and $\tau$.

It is a standard and easily verified fact that a Hamiltonian vector field $g$ with components $g_{j}$ on $\mathbb{C}^{2}$ has the form

$$
g_{j}=-2 i \frac{\partial H}{\partial \overline{z_{j}}}
$$

where we regard the Hamiltonian as a complex-valued (smooth) function of $z_{1}$ and $\overline{z_{1}}, z_{2}, \overline{z_{2}}, \lambda$ and $\tau$.

Putting these facts together, we find that

$$
\begin{aligned}
& g_{1}=-2 i H_{a} z_{1}+\ell\left[H_{d}-H_{c} i\right] \bar{z}_{1}^{\ell-1} z_{2}^{k} \\
& g_{2}=-2 i H_{b} z_{2}-k\left[H_{d}+H_{c} i\right] z_{1}^{\ell} \bar{z}_{2}^{k-1} .
\end{aligned}
$$

André Vanderbauwhede pointed out that these equations may be solved in a fashion similar to those obtained in the time-reversible case (15.8)-(15.11). Setting either $z_{1}=0$ or $z_{2}=0$ gives the $2 \pi / k$ and $2 \pi / \ell$ families of solutions as in Section 15 . We assume that $z_{1} \neq 0$ and $z_{2} \neq 0$. Phase-shift $\mathbf{S}^{1}$ symmetry allows us to set $z_{1}=x_{1}>0$. As in the time-reversible case, we solve for $\tau$ by using the implicit function theorem to solve $\operatorname{Im}\left(g_{1}\right)=0$. Indeed,

$$
\operatorname{Im}\left(g_{1}\right)=x_{1}\left[-2 H_{\mathrm{a}}+\ell x_{1}^{\ell-2} \operatorname{Im}\left(\left[H_{d}-i H_{c}\right] z_{2}^{k}\right)\right]=0
$$

can be solved for $\tau$ since $H_{a, \tau} \neq 0$ (recall $P_{1, \tau}^{i}(0) \neq 0$ ).

Next note that $\operatorname{Re}\left(g_{1}\right)=0$ and $\operatorname{Re}\left(g_{2}\right)=0$ are identical equations. We can write the two remaining equations as $\operatorname{Re}\left(g_{2} \overline{z_{2}}\right)=0$ and $\operatorname{Im}\left(g_{2} \overline{z_{2}}\right)=0$. Explicitly

$$
\begin{aligned}
& \operatorname{Re}\left(g_{2} \overline{z_{2}}\right)=-k x_{1}^{\ell} \operatorname{Re}\left(\left[H_{d}+i H_{c}\right]{\overline{z_{2}}}^{k}\right)=0 \\
& \operatorname{Im}\left(g_{2} \overline{z_{2}}\right)=-2 H_{b}\left|z_{2}\right|^{2}-k x_{1}^{\ell} \operatorname{Im}\left(\left[H_{d}+i H_{c}\right]{\overline{z_{2}}}^{k}\right)=0 .
\end{aligned}
$$

Equation (16.1) implies that

$$
\left(H_{d}+i H_{c}\right){\overline{z_{2}}}^{k}= \pm \sqrt{H_{d}^{2}+H_{c}^{2}}\left|z_{2}\right|^{k} i .
$$

Hence (16.2) may be rewritten as

$$
2 H_{b}\left|z_{2}\right|^{2} \pm k x_{1}^{\ell} \sqrt{H_{d}^{2}+H_{c}^{2}}\left|z_{2}\right|^{k}=0
$$

Assuming the nondegeneracy assumption (recall (R3) in Section 15)

$$
H_{d}(0) \neq 0 \quad \text { or } \quad H_{c}(0) \neq 0
$$

implies that $\sqrt{H_{d}^{2}+H_{c}^{2}}$ is smooth. Equation (16.3), upon division by $\left|z_{2}\right|$ or $\left|z_{2}\right|^{2}$ depending on whether $k=1$ or $k>1$, yields equations of the form (15.9) or (15.11). Once (16.3) is solved for $\left|z_{2}\right|$, the phase of $z_{2}$ is found using (16.1). 


\section{The case $k>1$}

We find solutions to (15.9) by using singularity theory to determine all small amplitude solutions. The singularity-theoretic results required in this context such as the 'tangent space constant' theorem and the universal unfolding theorem - are a consequence of general singularity theory results proved in Damon [1984].

The singularity theory calculations are most easily handled using the 'invariant' functions $r$ and $s$. (We omit the superscript $\epsilon$ in (15.9) in this discussion.) The changes of coordinates that we consider are given as follows. First, let

$$
u=x_{1}^{2} \quad \text { and } \quad v=x_{2}^{2} \text {, }
$$

and consider changes of coordinates in the domain of $p$ of the form

$$
\left(x_{1}, x_{2}\right) \mapsto\left(x_{1} X_{1}(u, v), x_{2} X_{2}(u, v)\right),
$$

where $X_{1}(0,0)>0$ and $X_{2}(0,0)>0$. These changes of coordinates preserve the $x_{1}$ and $x_{2}$ axes and the positive quadrant. We also allow multiplication of $p$ by functions of the form

$$
S(u, v)+R(u, v) x_{1}^{\ell-2} x_{2}^{k-2}
$$

where $S(0,0) \neq 0$. It is a simple exercise to check that these transformations preserve the form of $p$ and thus can be thought of as operations on the pair $(r(u, v), s(u, v))$, as is done for invariant functions in equivariant singularity theory.

In the context of the theory developed in Golubitsky and Schaeffer [1985], it is straightforward to compute the tangent space $T(p)$ for these coordinate changes. One finds that $T(p)$ is a module of function pairs in $\left(\mathcal{E}_{u, v}, \mathcal{M}_{u, v}\right)$ where $\mathcal{E}_{u, v}$ is the ring of germs of smooth, real-valued functions in the variables $u, v$ and $\mathcal{M}_{u, v} \subset \mathcal{E}_{u, v}$ is the maximal ideal generated by functions vanishing at the origin. This module has four generators:

$$
(r, s),\left(u^{\ell-2} v^{k-2} s, r\right),\left(2 u r_{u}, 2 u s_{u}+(\ell-2) s\right),\left(2 v r_{v}, 2 v s_{v}+(k-2) s\right) .
$$

Theorem 17.1 Assume that $\ell>k \geq 2$. If

$$
r_{u}, r_{v}, r_{u} s_{v}-r_{v} s_{u}
$$

are nonzero at the origin, then $p$ is equivalent to the normal form

$$
r(u, v)=u+\epsilon_{1} v, \quad s(u, v)=\epsilon_{2} u
$$

where $\epsilon_{1}=\operatorname{sgn}\left(r_{u}(0) r_{v}(0)\right)= \pm 1$ and $\epsilon_{2}=-\epsilon_{1} \operatorname{sgn}\left(r_{u} s_{v}-r_{v} s_{u}\right)= \pm 1$. This singularity has codimension one and a universal unfolding is

$$
r(u, v, \lambda)=u+\epsilon_{1} v+\lambda, \quad s(u, v)=\epsilon_{2} u .
$$

Proof Under the assumptions (17.4), $T(p)=\left(\mathcal{M}_{u, v}, \mathcal{M}_{u, v}\right)$. Hence terms of degree two and above in $r$ and $s$ may be eliminated by coordinate changes. Moreover, the 'tangent space constant' theorem allows us to transform $s$ to $s(u, v)=$ $\pm u$. Rescaling gives the appropriate normalizations to $r$.

We now discuss the zero sets of $p^{\epsilon}$ which has the form

$$
p^{e}=X_{1}^{2}+\epsilon_{1} X_{2}^{2}+\Lambda+\cdots,
$$




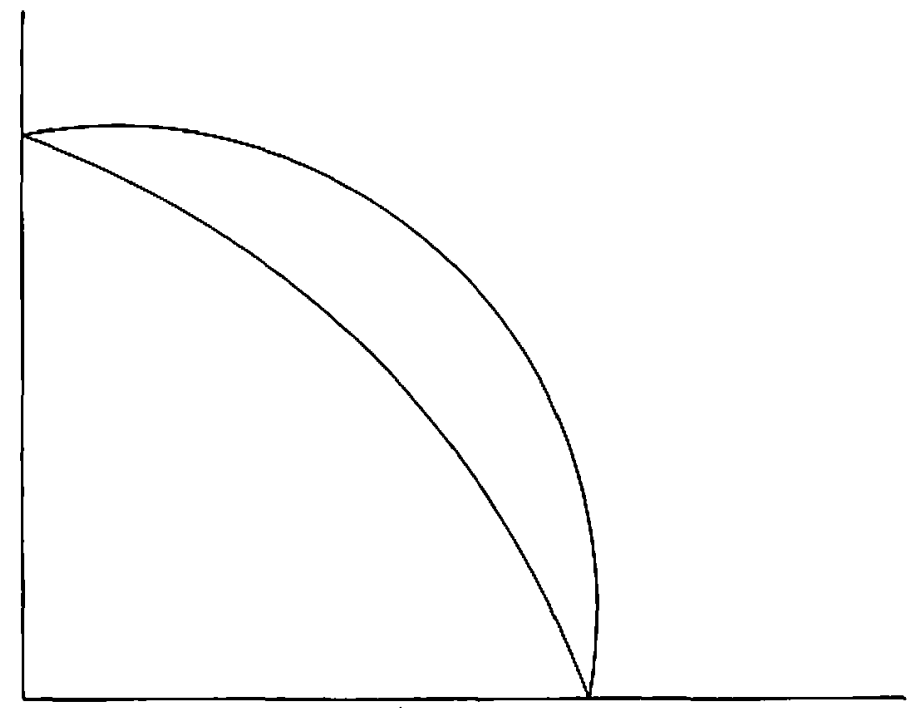

Figure $3 c>k \geq 2$ and $\epsilon_{1}=+1$. Equation $x_{1}^{2}+x_{2}^{2}+\lambda \pm x_{1}^{\ell} x_{2}^{k-2}=0$ when $\lambda<0$.

where $X\left(x_{1}, x_{2}, \lambda\right)=\left(X_{1}, X_{2}\right)$ and $\Lambda(\lambda)$ are invertible changes of coordinates. Note from (15.8) that $p^{+}=0$ and $p^{-}=0$ have the same solutions when either $x_{1}=0$ or $x_{2}=0$. Note also that the sign $\epsilon_{1}$ is the same for $p^{+}$and $p^{-}$since $\epsilon$ affects only higher order terms. Hence the zero set, restricted to $x_{1}>0, x_{2}>0$, is as shown in Figure 3 for $\epsilon_{1}=+1$ and $\lambda<0$. The figures for $\lambda<0$ and $\epsilon=-1$ may be drawn in a similar fashion.

\section{The case $k=1$}

Using singularity theory we determine all small amplitude solutions of (15.11). Since we do not need to preserve the first quadrant, we can allow a more general system of coordinate changes than we did in the previous section. The coordinate changes that we allow are:

$$
\left(x_{1}, x_{2}\right) \mapsto\left(x_{1} X_{1}(u, v), x_{2} X_{2}(u, v)+x_{1}^{\ell-2} X_{3}(u, v)\right)
$$

where $X_{2}(0,0) \neq 0$. These changes of coordinates still preserve the $x_{2}$ axis. Note that when $t=2$ we must require that $X_{3}(0,0)=0$ since we always assume that $s(0,0,0)=0$ in (15.11). (When $\ell=3$ we will enlarge the system of coordinate changes even further, see (18.10).) We also allow multiplication of $p$ by functions of the form

$$
S(u, v)+R(u, v) x_{1}^{i-2} x_{2}
$$

where $S(0,0) \neq 0$. It is an exercise to check that these transformations preserve the form of $p$ and hence can be thought of as operations on the pair $(r(u, v), s(u, v))$, as is done for invariant functions in equivariant singularity theory. However, it is not easy to write down the precise way that $(r, s)$ is transformed by these changes of coordinates.

It is also a standard exercise to compute the tangent space $T(p)$ for these coordinate changes. One finds that $T(p)$ is a module of function pairs in $\left(\mathcal{E}_{n . v}, \mathcal{M}_{n . v}\right)$ where $\mathcal{E}_{u, v}$ is the ring of germs of smooth, real-valued functions in the variables 
$u, v$ and $\mathcal{M}_{u, v} \subset \mathcal{E}_{u, v}$ is the maximal ideal generated by functions vanishing at the origin. This module has four generators:

$$
(r, s),\left(u^{\ell-2} s, v r\right),\left(2 u r_{u}, 2 u s_{u}+(\mathcal{E}-2) s\right),\left(2 v r_{v}+r, 2 v s_{v}\right),\left(2 u^{\ell-2} s_{v}, 2 v r_{v}+r\right)
$$

when $\ell>3$. When $\ell=2$ the last generator must be replaced by $u$ and $v$ times that generator.

As indicated, we distinguish three subcases: $\ell \geq 4, \ell=2$ and $\ell=3$. To complete the singularity theory for the case $\ell=3$ we must modify the discussion slightly.

Theorem 18.1 Assume that $\ell \geq 4$. If

$$
r_{u}, r_{v}, s_{u}, s_{v}, r_{u} s_{v}-3 r_{v} s_{u}
$$

are nonzero at the origin, then $p$ is equivalent to the normal form

$$
r(u, v)=u+\epsilon_{1} v, \quad s(u, v)=u
$$

where $\epsilon_{1}= \pm 1$. This singularity has codimension one and a universal unfolding is

$$
r(u, v, \lambda)=u+\epsilon_{1} v+\lambda, \quad s(u, v)=u .
$$

Proof Under assumptions (18.3), $\left(\mathcal{M}_{u, v}^{2}, \mathcal{M}_{u, v}^{2}\right) \subset \mathcal{M}_{u, v} T(p)$. The second generator in (18.2) is not used in this calculation. The standard 'tangent space constant' theorem implies that $p$ is 2-determined. Rescaling $x_{1}$ and $x_{2}$ and premultiplying $p$ by $S(0,0)$ produces the normal form (18.4). Since $x_{2} \neq 0$ when $k=1$ we may allow $X_{2}(0,0)$ to be nonzero - not just positive. Finally, we can now compute $T(p)$ exactly and see that the codimension of $(18.4)$ is one (the constant term in $r(0))$.

Theorem 18.2 Assume that $\ell=2$. If

$$
s_{u}, s_{v}
$$

are nonzero at the origin, then $p$ is equivalent to the normal form

$$
r(u, v)=0, \quad s(u, v)=\epsilon_{1} u+v
$$

where $\epsilon= \pm 1$. This singularity has codimension one and a universal unfolding is

$$
r(u, v, \lambda)=\lambda, \quad s(u, v)=\epsilon_{1} u+v .
$$

Proof Write $r=a u+b v$ and $s=c u+d v$. Under assumptions (18.6),

$$
T(p)=\left(\mathcal{M}_{u, v}, \mathcal{M}_{u, v}\right) \text {. }
$$

It follows that $p$ is 2-determined and we can assume $b=0$. Rescaling leads to the desired normal form. This singularity has codimension one with unfolding parameter $\lambda$ as indicated in (18.8).

Finally, we consider the case $\ell=3$. Now

$$
p\left(x_{1}, x_{2}\right)=r(u, v) x_{2}+s(u, v) x_{1}
$$


where $s(0,0)=0$. These $p$ 's are just the odd functions, that is, they satisfy

$$
p\left(-x_{1},-x_{2}\right)=-p\left(x_{1}, x_{2}\right)
$$

The appropriate coordinate changes are $X\left(x_{1}, x_{2}\right)$ where $X$ is odd. In addition, these coordinate changes must preserve the half-plane $x_{1} \geq 0$. The general such change of coordinates is:

$$
X\left(x_{1}, x_{2}\right)=\left(A x_{1}+B u x_{2}, C x_{1}+D x_{2}\right)
$$

where $A, B, C, D$ are functions of $u, v, A(0,0)>0$, and $D(0,0) \neq 0$. The contact equivalences in the range have the form

$$
S(u, v)+R(u, v) x_{1} x_{2}
$$

where $S(0,0) \neq 0$.

We now compute the tangent space $T(p)$ and find that it has six generators $\left(2 u r_{u}, 2 u s_{u}+s\right),\left(2 u^{2} s_{u}+u s, 2 u v r_{u}\right),\left(2 u s_{v}, 2 v r_{v}+r\right),\left(2 v r_{v}+r, 2 v s_{v}\right),(r, s),(u s, v r)$ corresponding to $A, B, C, D, S$ and $R$.

We next put the lowest order terms of $r, s$ into a normal form. Suppose that

$$
r(u, v)=a u+b v \quad \text { and } s(u, v)=c u+d v .
$$

Consider the change of coordinates

$$
X\left(x_{1}, x_{2}\right)=\left(A x_{1}, \mu A x_{1}+D x_{2}\right)
$$

where $A, D$, and $\mu$ are constants with $A>0, D \neq 0$. Then

$$
p\left(X\left(x_{1}, x_{2}\right)\right)=\hat{r}(u, v) x_{2}+\hat{s}(u, v) x_{1}
$$

where

$$
\begin{aligned}
& \hat{r}(u, v)=\left(a+2 d \mu+3 b \mu^{2}\right) A^{2} D u+b D^{3} v \\
& \hat{s}(u, v)=\left(c+a \mu+d \mu^{2}+b \mu^{3}\right) A^{3} u+(3 b \mu+d) A D^{2} v
\end{aligned}
$$

This calculation implies that.

$$
b \neq 0
$$

is required in a singularity of lowest codimension. Thus we assume that $r_{v}(0,0) \neq 0$ and set $\mu=-d / 3 b$. Next we assume that

$$
c+a \mu+d \mu^{2}+b \mu^{3} \neq 0
$$

which is equivalent to

$$
\alpha \equiv 2 d^{3}-9 a b d+27 b^{2} c \neq 0 .
$$

We now choose $A, D$ and $S(0,0)$ so that

$$
\hat{r}(u, v)=m u+v, \quad \hat{s}(u, v)=u,
$$

where

$$
m=3 \operatorname{sgn}(\alpha) \frac{3 a b-d^{2}}{\alpha^{2 / 3}}
$$

is a modal parameter. No further simplification of (18.13) is possible. 


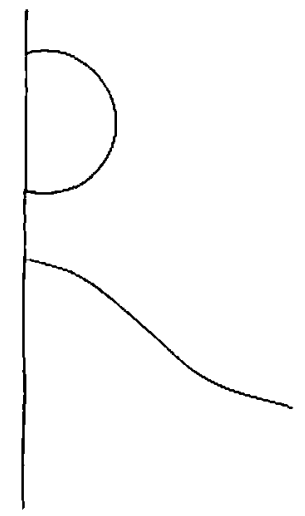

$\lambda<0$

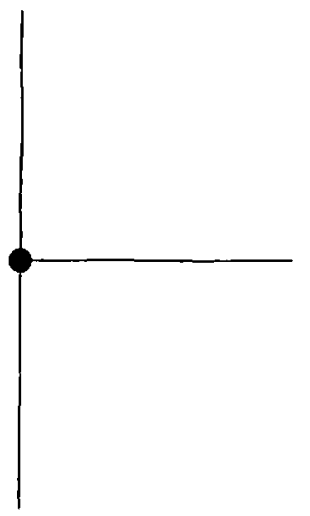

$\lambda=0$

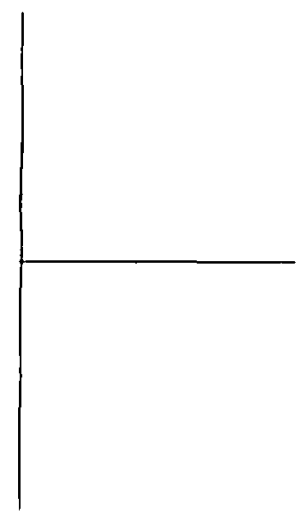

$\lambda>0$

Figure $4 k=1, \ell \geq 4$. Equation $x_{1}^{2} x_{2}+x_{2}^{3}+\lambda x_{2}+x_{1}^{\ell}=0$.

Theorem 18.3 Assume that $\ell=3$ and that (18.11) and (18.12) are satisfied. Then $p$ is equivalent to the normal form (18.13). This singularity has codimension two and a universal unfolding is

$$
r(u, v, \lambda)=m u+v+\lambda, \quad s(u, v)=u,
$$

where $m$ is a modal parameter.

Proof Using normal form (18.13) we can show that, independent of higher order terms,

$$
T(p)=\left(\mathcal{M}_{u, v}^{2}, \mathcal{M}_{u, v}^{2}\right) \oplus \mathbb{R}\{(2 m u, 3 u),(0, m u+3 v),(m u+3 v, 0)\}
$$

The 'langent space constant' theorem then says that we can eliminate higher order terms using a change of coordinates. The computation of the tangent space also shows that $p$ has codimension two - but one of those unfolding parameters is the modal parameter $m$ along which the germ $p$ has constant codimension.

\section{Bifurcation diagrams when $k=1$}

We now consider the solutions that may be derived from the normal forms of the previous theorems.

The case $\ell \geq 4$ In this case normal form (18.5) is

$$
x_{1}^{2} x_{2}+\epsilon_{1} x_{2}^{3}+\lambda x_{2}+x_{1}^{\prime}=0
$$

where $\epsilon_{1}= \pm 1$. This equation yields the pictures in Figures 4 and 5 .

The case $\ell=3$ In this case normal form (18.14) is

$$
x_{1}^{3}+m x_{1}^{2} x_{2}+x_{2}^{3}+\lambda x_{2}=0
$$

where $m \in \mathbb{R}$ is a modal parameter. The pictures are essentially the same as in the case $l \geq 4$. 


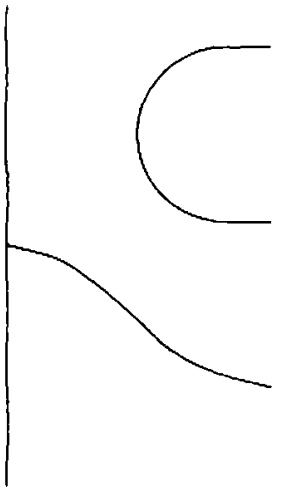

$\lambda<0$

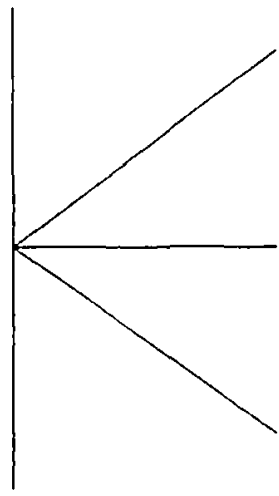

$\lambda=0$

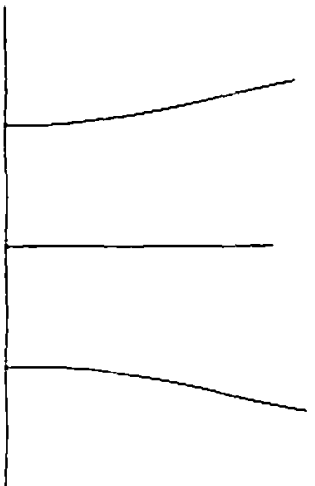

$\lambda>0$

Figure $5 k=1, \ell \geq 4$. Equation $x_{1}^{2} x_{2}-x_{2}^{3}+\lambda x_{2}+x_{1}^{f}=0$.

The case $\ell=2$ In this case normal form (18.8) is

$$
\epsilon_{1} x_{1}^{2}+x_{2}^{2}+\lambda=0
$$

where $\epsilon_{1}= \pm 1$. The case $\epsilon_{1}=+1$ and $\lambda<0$ is graphed in Figure 6 .

We conclude with a remark about the relation between the reversible and the Hamiltonian cases. Our analysis shows that the resulting bifurcations for the reversible and the Hamiltonian $k: \ell$ resonant cases are closely related. Arnol'd and others have raised the issue of why it is that the reversible and Hamiltonian cases so often lead to the same bifurcation diagrams? When the Hamiltonian problem satisfies condition (S), our results show that we end up with a Hamiltonian system with a Hamiltonian that is $\mathbf{S}^{1}$-invarinnt, and that in these cases, one has similar bifurcation diagrams. However, as we saw, the nonsemisimple Hamiltonian-Hopf case was rather different in the reversible and Hamiltonian cases. Another interesting link that might be useful for this general question is that, subject to some mild conditions, any Hamiltonian system can be realized as the $\mathbf{S}^{1}$ reduction of a reversible Hamiltonian one. The proof of this, whose details we do not give here, uses the Kaluza-Klein trick along with the theory of reduction of Lagrangian systems (see Marsden [1992] and Marsden and Scheurle [1993]). Thus. for example, the bifurcation of an equilibrium of a Hamiltonian system can be directly realized as the reduction of the bifurcation of a relative equilibrium in a reversible Hamiltonian system with $\mathbf{S}^{1}$-symmetry.

\section{Conclusions}

In this paper we have shown how singularity theory and the Liapunov-Schmidt procedure for systems with implicit constraints leads directly and simply to specific results for the bifurcation of periodic orbits. This method was applied to the Liapunov center theorem in the cases of systems with an integral, Hamiltonian systems and reversible ones. It was also applied to the Hamiltonian Hopf bifurcation, and to resonance bifurcations in both the Hamiltonian and reversible case's. In this way, we were able to recover the corresponding bifurcation results of Duistermaat [1983]. 


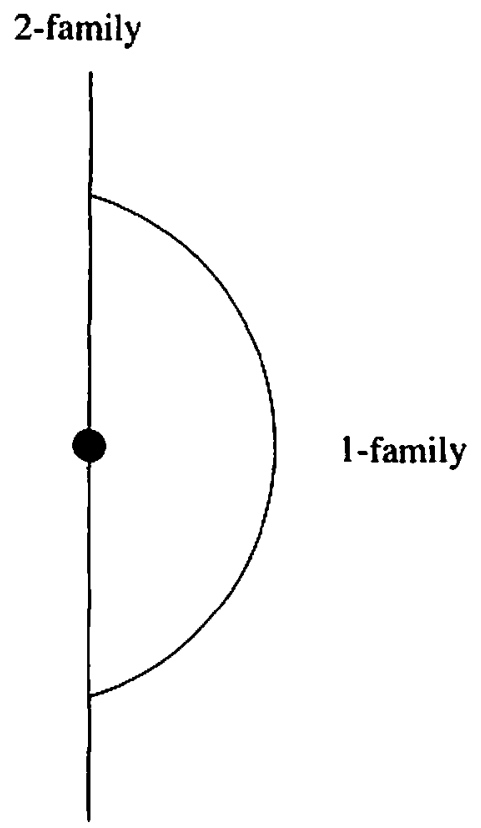

Figure $6 k=1, \ell=2$. Equation $x_{1}^{2}+x_{2}^{2}+\lambda=0$ when $\lambda<0$.

Sevryuk [1986] and Bridges [1990]. The method here, which looks for periodic orbits by applying the Liapunov-Schmidt procedure directly on loop spaces, has a number of technical advantages over normal form methods. We showed that the mapping that occurs in the procedure is a Hamiltonian vector field if a semisimple type of condition (S) is satisfied, and this is used to show that the bifurcation analysis of the reversible and Hamiltonian resonances are closely related. This condition holds for the Hamiltonian Liapunov center theorem and resonance bifurcations, but not for the Hamiltonian Hopf bifurcation, which is analyzed directly using the implicit constraint. In addition to analyzing stability, which we believe can be done by enhancing the present context using the techniques used for the Hopf bifurcation (see Golubitsky; Stewart and Schaeffer [1988]) combined with those of Montaldi, Roberts and Stewart [1988], there are many other problems to which the methods of this paper could in principle, be applied. For example, it would be interesting to analyze the case of passing resonances for Hamiltonian systems with symmetry, as in Dellnitz, Melbourne and Marsden [1992].

\section{References}

Abraham, R. and Marsden, J.E. [1978] Foundations of Mechanics, Second Edition, Addison-Wesley Publishing Co., Reading, Mass.

Abraham, R., Marsden, J.E. and Ratiu, T.S. [1988] Manifolds, Tensor Analysis, and Applications, Second Edition, Springer-Verlag, New York.

Alexander, J.C. and York, J.A. [1978] Global bifurcation of periodic orbits, Am. J. Math., 100, 263-292. 
Arnol'd, V.I. (ed.) [1988] Dynamical Systems III, Encyclopaedia of Math. Sciences, 3, Springer-Verlag, New York.

Bridges, T. [1990] Bifurcation of periodic solutions near a collision of eigenvalues of opposite signature, Math. Proc. Camb. Phil. Soc., 108, 575-601.

Chernoff, P.R. and Marsden, J.E. [1974] Properties of infinite dimensional Hamiltonian systems, Lecture Notes in Math., 425, Springer-Verlag, New York.

Cushman, R. and Rod, D.L. [1982] Reduction of the semisimple $1: 1$ resonance, Physica D, 6, 105-112.

Damon, J.N. [1984] The unfolding and determinacy theorems for subgroups of $\mathcal{A}$ and $\mathcal{K}$, Memoirs Vol. 50, No. 306, Amer. Math. Soc., Providence.

Dellnitz, M. and Melbourne, I. [1993] The equivariant Darboux theorem, Lectures in Appl. Math., 29, 163-169.

Dellnitz, M., Melbourne, I. and Marsden, J.E. [1992] Generic bifurcation of Hamiltonian vector fields with symmetry, Nonlinearity, 5, 979-996.

Duistermaat, H. [1983] Bifurcations of periodic solutions near equilibrium points of Hamiltonian systems, Lecture Notes in Math., 1057, Springer-Verlag, New York.

Duistermaat, H. [1984] Non-integrability of the $1: 2: 2$ resonance, Ergodic Theory and Dyn. Sys., 4, 553.

Golubitsky, M., Krupa, M. and Lim, C. [1991] Time reversibility and particle sedimentation, SIAM J. Appl. Math., 51, 49-72.

Golubitsky, M. and Langford, W. F. [1981] Classification of unfoldings of degenerate Hopf bifurcations, J. Diff. Eqns., 41, 375-415.

Golubitsky, M. and Schaeffer, D.G. [1985] Singularities and Groups in Bifurcation Theory: Vol. I, Applied Math. Sciences, 51, Springer-Verlag, New York.

Golubitsky, M. and Stewart, I. [1987] Generic bifurcation of Hamiltonian systems with symmetry, Physica D, 24, 391-405.

Golubitsky, M. and Stewart, I. [1993] An algebraic criterion for symmetric Hopf bifurcation, Proc. Roy. Soc. Lond. A, 440, 727-732.

Golubitsky, M., Stewart, I. and Schaeffer, D. [1988] Singularities and Groups in Bifurcation Theory: Vol. II, Applied Math. Sciences, 69, Springer-Verlag, New York.

Hale, J.K. [1969] Ordinary Differential Equations, Wiley, New York.

Haller, G. and Wiggins, S. [1992] Orbits homoclinic to resonances: the Hamiltonian case, Physica D, 66 298-346.

Haller, G. [1993], Multi-pulse Homoclinic Phenomena in Resonant Hamiltonian Systems, Thesis, Caltech.

Henrard, J. [1973] Lyapunov's center theorem for resonant equilibrium, J. Diff. Eq. 14, 431-441.

Hoveijn, I. and Verhulst, F. [1990] Chaos in the $1: 2: 3$ Hamiltonian normal form. Physica D, 44, 397. 
Iwai, T. [1985] On reduction of two degrees of freedom Hamiltonian systems by an $S^{1}$ action, and $S O_{0}[1,2]$ as a dynamical group, J. Math. Phys., 26, 885-893.

Kelley, A. [1967] The stable, center-stable, center, center-unstable, and unstable manifolds, J. Diff. Eq., 3, 546-570.

Knobloch, E., Mahalov, A. and Marsden, J.E. [1993] Normal Forms for threedimensional parametric instabilities in ideal hydrodynamics, Physica D, to appear.

Kummer, M. [1979] On resonant classical Hamiltonians with two degrees of freedom near an equilibrium point, Lecture Notes in Physics, 93, Springer-Verlag, 5775 .

Kummer, M. [1990] On resonant classical Hamiltonians with $n$ frequencies, J. Diff. Eq., 83, 220-243.

Liapunov, M.A. [1949] Problème générale de la stabilité du mouvement, Annals Math. Stud., 17, Princeton University Press.

Marsden, J.E. [1992], Lectures on Mechanics, London Math. Society Lecture Note Series, 174, Cambridge University Press, Cambridge.

Marsden, J.E. and McCracken, M. [1976] The Hopf bifurcation and its Applications, Applied Math. Sciences Series, 19, Springer-Verlag, New York.

Marsden, J.E. and Scheurle, J. [1993] The reduced Euler-Lagrange equations, Fields Institute Comm., 1, 139-164.

Meyer, K.R. and Palmore, J. [1970] A new class of periodic solutions in the restricted three-body problem, J. Diff. Eq., 8, 264-276.

Meyer, K.R. and Schmidt, D.S. [1971] Periodic orbits near $L_{4}$ for mass ratios near the critical mass ratio of Routh, Celest. Mech., 4, 99-109.

Montaldi, J.A., Roberts, R.M. and Stewart, I.N [1988] Periodic solutions near equilibria of symmetric Hamiltonian systems, Phil. Trans. R. Soc. Lond., A 325, 237-293.

O'Reilly, O., Malhotra, N.K. and Namachchivaya, N.S. [1993] Destabilization of the equilibria of reversible dynamical systems, preprint.

Palmore, J. [1969] Bridges and natural centers in the restricted three-body problem, Report. Univ. of Minnesota.

Rabinowitz, P.H. [1977] A bifurcation theorem for potential operators, J. of Funct. An., 25, $412 \backsim 424$.

Roberts, J.A.G. and Quispel, G.R.W. [1992] Chaos and time-reversal symmetry. Order and chaos in reversible dynamical systems, Phys. Reports, 216 63-177.

Roels, J. [1971a] An extension to resonant cases of Liapunov's theorem concerning the periodic solutions near a Hamiltonian equilibrium, J. Diff. Eq., 9, 300-324.

Roels. J. [1971b] Families of periodic solutions near a Hamiltonian equilibrium when the ratio of two eigenvalues is 3, J. Diff. Eq., 10, 431-447.

Sanders, J.A. and Verhulst, F. [1985] Averaging Methods in Nonlinear Dynamical Systems, Springer-Verlag, New York. 
Schmidt, D.S. [1973] Periodic solutions near a resonant equilibrium of a Hamiltonian system, Celest. Mech., 9, 81-103.

Schmidt, D.S. [1976a] Hopf's bifurcation theorem and the center theorem of Liapunov, Celest. Mech., 9, 81-103.

Schmidt, D.S. [1976b] Hopf's bifurcation theorem and the center theorem of Liapunov with resonance cases, Section 3C of Marsden and McCracken [1976].

Schmidt, D.S. and Sweet, D. [1973] A unifying theory in determining periodic families for Hamiltonian systems at resonance, J. Diff. Eq., 14, 597-609.

Sevryuk, M.B. [1986] Reversible Systems, Lect ure Notes in Math., 1211, SpringerVerlag, Berlin.

Sweet, D. [1973] Periodic solutions for dynamical systems possessing a first integral in the resonance case, J. Diff. Eq., 14, 171-183.

Vanderbauwhede, A. [1982a] Local Bifurcation and Symmetry, Research Notes in Math., 75, Pitman, Boston.

Vanderbauwhede, A. [1982b] Families of periodic solutions for autonomous systems; in Dynamical Systems II (A. Bednarek and L. Cesari, eds), Academic Press, $427-446$.

Vanderbauwhede, A. [1990] Hopf bifurcation for equivariant conservative and timereversible systems, Proc. Roy. Soc., Edinburgh, 116A, 103-128.

Vanderbauwhede, A. and van der Meer, J.C. [1994] A general reduction method for periodic solutions near equilibria in Hamiltonian systems, Fields Institute Comm., to appear.

van der Meer, J.C. [1985] The Hamiltonian-Hopf Bifurcation. Lecture Notes in Math., 1160, Springer-Verlag, Berlin.

Weinstein, A. [1978] Bifurcations and Hamilton's principle, Math. Zeit., 159, 235248. 